TRANSACTIONS OF THE

AMERICAN MATHEMATICAL SOCIETY

Volume 357, Number 7, Pages 2771-2798

S 0002-9947(04)03583-4

Article electronically published on September 2, 2004

\title{
COMMUTATIVE IDEAL THEORY WITHOUT FINITENESS CONDITIONS: PRIMAL IDEALS
}

\author{
LASZLO FUCHS, WILLIAM HEINZER, AND BRUCE OLBERDING
}

\begin{abstract}
Our goal is to establish an efficient decomposition of an ideal $A$ of a commutative ring $R$ as an intersection of primal ideals. We prove the existence of a canonical primal decomposition: $A=\bigcap_{P \in \mathcal{X}_{A}} A_{(P)}$, where the $A_{(P)}$ are isolated components of $A$ that are primal ideals having distinct and incomparable adjoint primes $P$. For this purpose we define the set $\operatorname{Ass}(A)$ of associated primes of the ideal $A$ to be those defined and studied by Krull. We determine conditions for the canonical primal decomposition to be irredundant, or residually maximal, or the unique representation of $A$ as an irredundant intersection of isolated components of $A$. Using our canonical primal decomposition, we obtain an affirmative answer to a question raised by Fuchs, and also prove for $P \in$ Spec $R$ that an ideal $A \subseteq P$ is an intersection of $P$-primal ideals if and only if the elements of $R \backslash P$ are prime to $A$. We prove that the following conditions are equivalent: (i) the ring $R$ is arithmetical, (ii) every primal ideal of $R$ is irreducible, (iii) each proper ideal of $R$ is an intersection of its irreducible isolated components. We classify the rings for which the canonical primal decomposition of each proper ideal is an irredundant decomposition of irreducible ideals as precisely the arithmetical rings with Noetherian maximal spectrum. In particular, the integral domains having these equivalent properties are the Prüfer domains possessing a certain property.
\end{abstract}

\section{INTRODUCTION}

In her seminal paper [27, Emmy Noether proved that in a commutative ring satisfying the ascending chain condition on ideals every ideal is the intersection of a finite number of irreducible ideals, and the irreducible ideals are primary ideals. She went on to establish several intersection decompositions, one of which featured primary ideal components with distinct radicals.

Among rings without the ascending chain condition, the rings in which such a decomposition holds for all ideals (called Laskerian rings) are few and far between (see, e.g., Heinzer and Lantz [13]). One reason for this is that irreducible ideals need not be primary. Thus, if we wish to obtain analogous decompositions in general rings, then we have to replace primary ideals by more general ideals. Natural candidates are the primal ideals introduced in Fuchs [5], where it is shown that every ideal is the intersection of (in general infinitely many) irreducible ideals,

Received by the editors January 2, 2003 and, in revised form, November 4, 2003.

2000 Mathematics Subject Classification. Primary 13A15, 13F05.

Key words and phrases. Primal ideal, associated prime, arithmetical ring, Prüfer domain.

(C)2004 American Mathematical Society 
and these ideals are primal ideals. This primal decomposition of an ideal gives components whose structure we know, but the components are not closely tied to the ideal and there is no indication of how many and what kind of primal ideals are needed for the intersection. It is also possible to represent an ideal as the intersection of its isolated components. In this case the components are closely tied to the ideal, but the structure of the components is not known. In general, the isolated components of an ideal are not primal ideals and there are almost always superfluous components.

Our main purpose here is to establish a more efficient decomposition of an ideal into the intersection of primal ideals. We show in Theorem 3.5 that if we take just those components that appear in both decompositions, then we can still represent the ideal even with the minimal isolated primal components. The existence of such decompositions in the Noetherian case was established in Fuchs [5]. In Theorem 3.5 we achieve a canonical primal decomposition (that is often irredundant), where the primal components belong to distinct, incomparable primes and where these distinct primes are the maximal primes in the set of associated primes of $A$; here we are using associated primes in the sense defined by Krull [19] page 742]. Also see [1, page 253]. With this definition of associated primes, maximal associated primes of $A$ exist and every associated prime is contained in a maximal associated prime.

Krull [20, page 16] mentions that it was an undecided problem whether or not (phrased in our terminology) the principal component $A_{(P)}$ (for $P$ a maximal prime divisor of $A$ ) is $P$-primal. Nagata in [24] and Gilmer in [8] gave a negative answer, but the full answer is given in Theorem 3.4: the isolated $P$-component of $A$ is $P$-primal if and only if $P$ is a Krull associated prime of $A$.

We establish in Corollary 3.6 an affirmative answer to the question raised in [5. page 3] of whether for a maximal prime divisor $P$ of an ideal $A$ the principal $P$-component $A_{(P)}$ is equal to the intersection of all primal ideals that contain $A$ and have their associated adjoint primes contained in $P$. Indeed, Proposition 3.7 implies that $A_{(P)}$ is an intersection of $P$-primal ideals. In Example 3.8 we present an example where the ideal $A$ has precisely two maximal prime divisors and where the principal component with respect to each of these maximal prime divisors is not a primal ideal.

We prove in Theorem 4.2 that under certain additional hypothesis the canonical primal decomposition of Theorem 3.5 is irredundant. We establish in Proposition 4.3 under certain conditions that a stronger form of irredundancy holds: namely, the canonical primal decomposition of Theorem 3.5 is residually maximal in the sense that no component can be replaced by any of its proper residuals. For $P$ a maximal associated prime of the ideal $A$, we give in Theorem 4.6 necessary and sufficient conditions in order that the isolated component $A_{(P)}$ appear in every representation of $A$ as an intersection of primal isolated components of $A$.

In Section 5 we consider irredundant decompositions into irreducible ideals. If an ideal $A$ has a representation as an irredundant intersection of irreducible isolated components, we prove in Theorem 5.1 that this intersection is residually maximal and is the unique irredundant decomposition of $A$ into irreducible isolated components of $A$. Example 5.4 shows there are limitations as to any sharpening of Theorem 5.1. In Theorem 5.8 we prove that a ring $R$ is an arithmetical ring if and only if each proper ideal of $R$ can be represented as an intersection of irreducible 
isolated components. Indeed, if $R$ is an arithmetical ring and $A$ can be represented as an irredundant intersection of irreducible ideals, then this is the unique representation of $A$ as an irredundant intersection of irreducible ideals (Corollary 5.6).

We also consider in Section 5 the question of what rings $R$ have the property that the canonical primal decomposition of each proper ideal $A$ of $R$ is an irredundant representation of $A$ as an intersection of irreducible isolated components. We classify in Theorem 5.14 the rings $R$ having this property as the arithmetical rings with Noetherian maximal spectrum. In particular, the integral domains having this property are precisely the Prüfer domains that satisfy condition (\#\#) (defined in the paragraph before Lemma 5.13).

\section{Preliminaries}

Throughout, $R$ will denote a commutative ring with 1 . For ideals $I, J$ of $R$, the residual $I: J$ is defined as usual by

$$
I: J=\{x \in R: x J \subseteq I\} .
$$

For an ideal $A$ and for a prime ideal $P$ of $R$, we use the notation

$$
A_{(P)}=\{x \in R: s x \in A \text { for some } s \in R \backslash P\}=\bigcup_{s \in R \backslash P} A: s
$$

to denote the isolated $P$-component (isoliertes Komponentenideal) of $A$ in the sense of Krull [20 page 16]. It will be useful to observe that $x \in A_{(P)}$ if and only if $A: x \nsubseteq P$. If $R$ is a domain, then $A_{(P)}=A R_{P} \cap R$, where $R_{P}$ denotes the localization of $R$ at $P$.

Evidently, $A_{(P)} \subseteq P$ for every prime $P$ with $A \subseteq P$. If $P$ is a minimal prime of $A$, then $A_{(P)}$ is known to be a $P$-primary ideal: the isolated $P$-primary component of $A$.

Lemma 1.1. For every ideal $A$, prime ideal $P$ and element $x \in R$, we have

$$
(A: x)_{(P)}=A_{(P)}: x
$$

Proof. Evidently, $y \in(A: x)_{(P)} \Leftrightarrow \exists c \notin P$ such that $c y \in A: x \Leftrightarrow \exists c \notin P$ with $c x y \in A \Leftrightarrow x y \in A_{(P)} \Leftrightarrow y \in A_{(P)}: x$.

An element $x \in R$ is called non-prime to $A$ if $A \subset A: x$ (proper inclusion), i.e., there exists an element $c \in R \backslash A$ such that $c x \in A$. Evidently, the set $S(A)$ of elements non-prime to $A$ corresponds to the set of zero-divisors in the factor ring $R / A$; it is the set union of prime ideals: the maximal prime divisors of $A$.

An ideal $A$ is said to be primal (see [5]) if $S(A)$ itself is an ideal, i.e., if the elements of $R$ that are not prime to $A$ form an ideal $P$; this ideal is then a prime ideal, called the adjoint ideal of $A$. In this case, we also say that $A$ is a $P$-primal ideal. An ideal $A$ is primal if and only if the sum of any two ring elements non-prime to $A$ is again non-prime to $A$.

Irreducible ideals are in general not primary (for a necessary and sufficient condition, see Fuchs 4]). Primal ideals have the advantage over primary ideals that the following is true without any chain condition:

Lemma 1.2 (Fuchs [5]). (a) Irreducible ideals are primal.

(b) Every ideal is the intersection of primal ideals. 
We use the following result.

Lemma 1.3. If $A$ is a P-primal ideal, then

(i) $A_{(Q)}=A$ if $Q$ is a prime containing $P$;

(ii) $A_{(Q)} \supset A$ (proper inclusion) if $Q$ is a prime not containing $P$; and

(iii) if $A_{(Q)}$ is a $Q$-primal ideal for some prime $Q$ containing $A$, then $Q \subseteq P$.

Proof. (i) To show that $A_{(Q)} \subseteq A$, observe that $x \in A_{(Q)}$ means $c x \in A$ for some $c \in R \backslash Q$. But if $A$ is $P$-primal, then $c$ is prime to $A$, so $x \in A$.

(ii) There exists an $x \in P \backslash Q$. Such an $x$ is not prime to $A$, so for some $y \notin A$ we have $x y \in A$. Then $y \in A_{(Q)} \backslash A$.

(iii) By assumption, $Q=\bigcup_{x \in R \backslash A_{(Q)}} A_{(Q)}: x$. If $Q \nsubseteq \nsubseteq P$, then there exists $q \in Q \backslash P$ such that $q x \in A_{(Q)}$ for some $x \in R \backslash A_{(Q)}$. Thus there exists $c \in R \backslash Q$ such that $c q x \in A$. Now $q \notin P$, so since $A=A_{(P)}, c x \in A$. However, $c \notin Q$, so $x \in A_{(Q)}$, contrary to assumption. We conclude that $Q \subseteq P$.

Primal ideals behave similarly to primary ideals, but there are notable differences. For instance, the intersection of two primal ideals with the same adjoint prime need not be primal again. A counterexample is the principal ideal $x y R$ in the polynomial ring $R=F[x, y]$, where $x$ and $y$ are indeterminates over a field $F$. The ideal $x y R$ is not primal ( $x, y$ are not prime to $x y R$, but $x-y$ is), though it is the intersection of the $(x, y) R$-primal ideals $\left(x^{2}, x y\right) R$ and $\left(x y, y^{2}\right) R$. However, if we have a reduced intersection (i.e., none of the components may be replaced by a larger ideal without changing the intersection), then we can claim:

Lemma 1.4 (Fuchs [5]). A reduced intersection $A=A_{1} \cap \cdots \cap A_{n}$ of primal ideals $A_{i}$ with adjoint primes $P_{i}$ is again primal if and only if there is a unique maximal member in the set $\left\{P_{1}, \ldots, P_{n}\right\}$. This prime is then the adjoint prime to $A$.

Recall that the closed sets of the prime spectrum $\operatorname{Spec} R$ of the ring $R$ in the Zariski topology are the sets $V(I)=\{P \in \operatorname{Spec} R: P \supseteq I\}$ with $I$ ranging over the set of ideals of $R$. Spec $R$ is called Noetherian if the closed subsets in the Zariski topology satisfy the descending chain condition, or equivalently, if the radical ideals of $R$ satisfy the ascending chain condition. The maximal spectrum of $R$ is the set Max $R$ of maximal ideals of $R$ with the subspace topology from $\operatorname{Spec} R$. We say that $R$ has Noetherian maximal spectrum if the closed subsets of Max $R$ satisfy the descending chain condition, or equivalently, if the J-radical ideals of $R$ satisfy the ascending chain condition, where an ideal is a J-radical ideal if it is an intersection of maximal ideals.

Some of the following characterizations of rings with Noetherian prime spectra will be needed in our discussions.

Lemma 1.5 (Mori [23] or Ohm and Pendelton [28]). For a commutative ring $R$ the following conditions are equivalent:

(i) $\operatorname{Spec} R$ is Noetherian.

(ii) $R$ has the maximum condition on radical ideals.

(iii) The ascending chain condition holds for prime ideals, and each ideal of $R$ has only finitely many minimal prime ideals. 
We will pay special attention to the class of arithmetical rings. In addition to providing an interesting contrast to the theory of primal decompositions in Noetherian rings, Theorem 1.8 and the results of Section 5 show that this class of rings arises naturally in our context.

Recall that a ring $R$ is a chained ring or valuation ring if the ideals of $R$ are linearly ordered with respect to inclusion; and $R$ is an arithmetical ring if $R_{M}$ is a valuation ring for each maximal ideal $M$ of $R$. An integral domain is an arithmetical ring if and only if it is a Prüfer domain. Among several results that are relevant to our study is the theorem of Jensen that primary ideals of an arithmetical ring are irreducible and that a Prüfer domain $R$ has the property that each ideal of $R$ with prime radical is irreducible if and only if each nonzero prime ideal of $R$ is contained in a unique maximal ideal [17, Theorem 6]. It is readily seen that a ring $R$ is an arithmetical ring if and only if for each proper ideal $A$ of $R, A_{M}$ is an irreducible ideal of $R_{M}$ for every maximal ideal $M$ of $R$ containing $A$.

Remark 1.6. Let $A$ be an ideal of a ring $R$ and let $P \in \operatorname{Spec} R$. The ideal $A_{P}$ is irreducible in $R_{P}$ if and only if $A_{(P)}$ is irreducible in $R$. This statement is clear in view of the following facts: (i) finite intersections of ideals of $R$ behave well with respect to the map $R \rightarrow R_{P}$, (ii) every ideal of $R_{P}$ comes from an ideal of $R$, and (iii) $A_{(P)}$ is the preimage in $R$ of the ideal $A_{P}$ of $R_{P}$.

From this observation we deduce the characterization: $A$ ring $R$ is arithmetical if and only if for all ideals $A$ of $R, A_{(M)}$ is an irreducible ideal for all maximal ideals $M$ that contain $A$. For if $R$ is arithmetical, then every ideal of $R_{M}, M$ a maximal ideal of $R$, is irreducible, so if $A$ is an ideal of $R$ contained in $M, A_{M}$, and hence $A_{(M)}$, are irreducible ideals of $R_{M}$ and $R$, respectively. Conversely, as noted in the paragraph before Remark 1.6 to prove $R$ is arithmetical it is enough to show for every ideal $A$ and maximal ideal $M$ containing $A$ that $A_{M}$ is an irreducible ideal. This is indeed the case, since by assumption $A_{(M)}$ is irreducible and we have from the above observation that $A_{M}$ is an irreducible ideal of $R_{M}$.

Lemma 1.7. Let $A$ be a finitely generated ideal contained in a prime ideal $P$ of the ring $R$. If $A_{P} \neq 0$, then $(A P)_{(P)}$ is a $P$-primal ideal of $R$.

Proof. Set $B=A P$. Clearly, the elements of $R$ not prime to $B_{(P)}$ are contained in $P$, so to show $B_{(P)}$ is a $P$-primal ideal of $R$, it suffices to prove that the elements of $P$ are not prime to $B_{(P)}$. Since $A$ is finitely generated and $A_{P} \neq 0$, Nakayama's Lemma implies that $B_{P} \neq A_{P}$, and it follows that $B_{(P)} \neq A_{(P)}$. Thus $B_{(P)} \subset$ $A_{(P)} \subseteq B_{(P)}: P$, so there exists $y \in R \backslash B_{(P)}$ such that $y P \subseteq B_{(P)}$. This proves that the elements of $P$ are not prime to $B_{(P)}$ and $B_{(P)}$ is a $P$-primal ideal.

Theorem 1.8. The following statements are equivalent for a ring $R$.

(i) $R$ is an arithmetical ring.

(ii) Every primal ideal of $R$ is irreducible.

Proof. (i) $\Rightarrow$ (ii). The implication (i) $\Rightarrow$ (ii) is proved in [7] for the case where $R$ is a domain. Allowing zero divisors requires a simple modification of this argument. If $A$ is a primal ideal, then $A=A_{(P)}$, where $P$ is the adjoint prime of $A$. By (i), $A_{P}$, and hence $A_{(P)}$ (Remark [1.6), are irreducible ideals, and this proves (ii).

(ii) $\Rightarrow$ (i) We use the following observation: ( $\dagger$ ) Let $I=(a, b) R$ be a finitely generated ideal contained in a maximal ideal $M$ of $R$. If $I_{M} \neq 0$ and $I M_{M}$ is an irreducible ideal of $R_{M}$, then $I_{M}=R_{M}$ a or $I_{M}=R_{M} b$. 
Indeed, the $R / M$-vector space $I_{M} / I M_{M}$ is finite-dimensional. Since $I M_{M}$ is irreducible, it follows that $I_{M} / I M_{M} \cong R / M$. In particular, $I_{M}=a R_{M}+I M_{M}$ or $I_{M}=b R_{M}+I M_{M}$. By Nakayama's Lemma, $I_{M}=a R_{M}$ or $I_{M}=b R_{M}$.

To prove (i), it suffices to show, for each maximal ideal $M$ of $R$ and $a, b \in M$, that either $R_{M} a \subseteq R_{M} b$ or $R_{M} b \subseteq R_{M} a$, for this implies that $R_{M}$ is a valuation ring. We may also assume that $I=(a, b) R$ is such that $I_{M} \neq 0$. By Lemma 1.7 $(I M)_{(M)}$ is an $M$-primal ideal, so by (ii) and Remark 1.6 $I M R_{M}$ is an irreducible ideal of $R_{M}$. Thus by the observation ( $\dagger$ ), either $R_{M} a \subseteq R_{M} b$ or $R_{M} b \subseteq R_{M} a$.

\section{Associated PRIMes}

We start with the study of primes associated to an ideal. There are at least six inequivalent notions of associated primes in the literature, all of which are equivalent in the Noetherian case. (See Heinzer and Ohm 14 and Iroz and Rush [16], for example.)

For our purposes, the most useful concept of associated primes is one introduced by Krull [19, page 742]. Let $A$ be a proper ideal of the ring $R$. Following [16, we define a prime ideal $P$ of a ring $R$ to be a Krull associated prime of the ideal $A$ if for every element $x \in P$, there exists $y \in R$ such that $x \in A: y \subseteq P$. A prime $P$ of $R$ is called a weak-Bourbaki associated prime to $A$ if it is a minimal prime divisor of $A: x$ for some $x \in R \backslash A$. Following [14 page 279], we call $P$ a Zariski-Samuel associated prime of $A$ if $P=\sqrt{A: x}$ for some $x \in R$. (This differs slightly from the terminology of [31, where $P$ is said to be a Zariski-Samuel associated prime of $A$ if $A: x$ is $P$-primary for some $x \in R$. Of course if $A: x$ is $P$-primary, then $P$ is

a Zariski-Samuel associated prime in our sense.) It is clear that a Zariski-Samuel associated prime is a weak-Bourbaki associated prime.

Lemma 2.1. Let $A$ be a proper ideal of the ring $R$. Every weak-Bourbaki associated prime of $A$ is a Krull associated prime of $A$. A prime ideal $Q$ of $R$ is a Krull associated prime of $A$ if and only if $Q$ is a set-theoretic union of weak-Bourbaki associated primes of $A$.

Proof. Let $P$ be a weak-Bourbaki associated prime of $A$. Then $P$ is a minimal prime of $A: x$ for some $x \notin A$. It follows that the ideal $(A: x)_{(P)}$ is $P$-primary. Thus given $u \in P$, there is a smallest integer $k \geq 1$ such that $u^{k} \in(A: x)_{(P)}$. Hence $u^{k} v \in A: x$ for some $v \notin P$. Evidently, $u \in A: x u^{k-1} v$. If $A: x u^{k-1} v \subseteq P$ were not true, then we could find a $w \notin P$ with $w \in A: x u^{k-1} v$. But then $u^{k-1} v w \in A: x$, and $u^{k-1} \in(A: x)_{(P)}$ is impossible. Thus $A: x u^{k-1} v \subseteq P$, indeed. (See also [21, Theorem 1] or [16, page 346].) It follows that a prime ideal $Q$ of $R$ is a Krull associated prime of $A$ if it is a set-theoretic union of weak-Bourbaki associated primes of $A$. The converse is clear, since $x \in A: y \subseteq Q$ implies that $x$ is contained in every minimal prime of $A: y$.

It is obvious that every proper ideal of the ring has weak-Bourbaki associated primes; hence every proper ideal possesses at least one Krull associated prime. However, this is not the case for Zariski-Samuel associated primes: Nakano [26] gives an example of a Prüfer domain in which finitely generated ideals do not admit any Zariski-Samuel associated prime.

We are convinced that the notion of a Krull associated prime is more useful (and perhaps more natural) than any of the other definitions proposed in the literature, because besides being equivalent to the classical notion of associated prime 
in Noetherian rings, it has the following pleasant properties: 1) the set union of the Krull associated primes of an ideal $A$ is the set of elements non-prime to $A$ (so there is a sufficient supply of them); 2) every Krull associated prime is contained in a maximal Krull associated prime; 3) Krull associated primes are preserved under localization; and 4) they can be recognized by a distinguished property of the corresponding isolated components of $A$ (see Theorem 3.4).

For an ideal $A$ of $R$ we follow [2, page 289] and define $\operatorname{Ass}_{f}(A)$ to be the set of weak-Bourbaki associated primes of $A$. We reserve the notation $\operatorname{Ass}(A)$ for the set of Krull associated primes of $A$, that is,

$$
\operatorname{Ass}(A)=\left\{P: P \text { is a prime that is the set union of primes in } \operatorname{Ass}_{f}(A)\right\} .
$$

Finally, let

$$
\mathcal{X}_{A}=\{P: P \text { is a maximal member of } \operatorname{Ass}(A)\} .
$$

That there is a sufficient supply of primes in $\mathcal{X}_{A}$ (and hence in $\operatorname{Ass}(A)$ ) is shown by the next lemma (cf. [2, Exercise 17(b), page 289]).

Lemma 2.2. Let $A$ be a proper ideal of the ring $R$ and let $S(A)$ denote the set of elements of $R$ that are non-prime to $A$. Then

$$
S(A)=\bigcup_{P \in \operatorname{Ass}_{f}(A)} P=\bigcup_{P \in \operatorname{Ass}(A)} P=\bigcup_{P \in \mathcal{X}_{A}} P .
$$

Proof. The last two equalities being obvious, it suffices to verify the first. Let $u \in P$ for some $P \in \operatorname{Ass}_{f}(A)$. Since $P$ is a minimal prime of $A: x$ for some $x \in R \backslash A$, and $(A: x)_{(P)}=A_{(P)}: x$ is a $P$-primary ideal, there is an integer $k>0$ such that $u^{k} \in A_{(P)}: x$. Thus $s u^{k} x \in A$ for some $s \notin P$. But $A: x \subseteq P$ implies $s x \notin A$, so $u^{k} \in S(A)$, and hence $u \in S(A)$ as well.

On the other hand, if $u \in S(A)$, then there is a $v \notin A$ such that $u \in A: v$. If $P$ is a minimal prime divisor of $A: v$, then $u \in A: v \subseteq P \in \operatorname{Ass}_{f}(A)$.

The maximal prime divisors of $A$ (i.e., the ideals maximal in the set $S(A)$ ) need not belong to $\operatorname{Ass}(A)$. This situation is illustrated by examples given by Nagata [24], by Gilmer [8], and by Example 3.8 presented below. However, we have

Corollary 2.3. If $A$ is a primal ideal of $R$, then its adjoint prime ideal $P$ is a Krull associated prime of $A$.

Proof. By Lemma 2.2, $P=S(A)$ is as stated.

If $A$ is $P$-primal, then $P$ is a Zariski-Samuel prime of $A$ if and only if $P$ is a weak-Bourbaki prime of $A$. For it is always true that Zariski-Samuel implies weak-Bourbaki. On the other hand, if $x \in R \backslash A$, then every minimal prime of $A: x$ consists of elements non-prime to $A$. Therefore if $A$ is $P$-primal, then every minimal prime of $A: x$ is contained in $P$. Hence $P$ is a minimal prime of $A: x$ implies $P=\sqrt{A: x}$ and $P$ is a Zariski-Samuel prime of $A$.

As far as the behavior of associated primes under localization is concerned, we have the most satisfactory result (cf. [2, Exercise 17(d), page 289]):

Lemma 2.4. Let $A$ be an ideal of the ring $R$ and let $P$ be a prime ideal of $R$ containing $A$.

(i) The following statements are equivalent for $P$ :

(a) $P \in \operatorname{Ass}_{f}(A)$. 
(b) $P_{M} \in \operatorname{Ass}_{f}\left(A_{M}\right)$ for every maximal ideal $M$ of $R$ containing $P$.

(c) $P_{M} \in \operatorname{Ass}_{f}\left(A_{M}\right)$ for some maximal ideal $M$ of $R$ containing $P$.

(ii) The following statements are equivalent for $P$ :

(a) $P \in \operatorname{Ass}(A)$.

(b) $P_{M} \in \operatorname{Ass}\left(A_{M}\right)$ for every maximal ideal $M$ of $R$ containing $P$.

(c) $P_{M} \in \operatorname{Ass}\left(A_{M}\right)$ for some maximal ideal $M$ of $R$ containing $P$.

Proof. (i) To see that (a) implies (b), suppose $P$ is a minimal prime over $A: r$ for some $r \in R \backslash A$, and let $M$ be a maximal ideal of $R$ containing $P$. There exists a prime ideal $Q$ of $R$ such that $Q_{M}$ is minimal over $A_{M}: r$, so $A_{M}: r \subseteq Q_{M} \subseteq P_{M}$. In this case, $A: r \subseteq Q \subseteq P$, so $Q=P$. This proves (a) implies (b). That (b) implies (c) is clear, so to prove that (c) implies (a), suppose there is a maximal ideal $M$ of $R$ such that $P_{M}$ is minimal over $A_{M}: s$ for some $s \in R \backslash A_{M}$. Then $A: s \subseteq P$, and there exists a minimal prime $Q$ of $A: s$ such that $A: s \subseteq Q \subseteq P$. This implies $A_{M}: s \subseteq Q_{M} \subseteq P_{M}$, so $Q_{M}=P_{M}$ and $Q=P$.

(ii) If $P \in \operatorname{Ass}(A)$, then $P$ is the union of weak-Bourbaki primes $Q$ of $A$. Let $M$ be a maximal ideal of $R$ that contains $P$. By (i), $Q_{M}$ is a weak-Bourbaki prime of $A_{M}$, so $P_{M}$ is the union of weak-Bourbaki primes $Q_{M}$ of $A_{M}$. This proves (a) implies (b). The assertion that (b) implies (c) is clear. To complete the proof, we verify (c) implies (a). Suppose $P_{M} \in \operatorname{Ass}\left(A_{M}\right)$ for some maximal ideal $M$ containing $P$. Then $P_{M}$ is the union of weak-Bourbaki primes $Q_{M}$ of $A_{M}$. By (i), the preimage $Q$ in $R$ of each $Q_{M}$ is a weak-Bourbaki prime of $A$, so $P$ is the union of weak-Bourbaki primes of $A$.

With a hypothesis of Noetherian spectrum we can say more about the primes in $\operatorname{Ass}(A)$ and about the set $\mathcal{X}_{A}$. However, as Example 3.8 below illustrates, it is possible even in a ring with Noetherian spectrum for there to exist an ideal $A$ having a maximal prime divisor $P$ such that $P \notin \operatorname{Ass}(A)$. (This cannot happen if the ring is Noetherian.)

Lemma 2.5. Suppose that $A$ is a proper ideal of the ring $R$ and the spectrum of $R / A$ is Noetherian.

(i) All the ideals in $\operatorname{Ass}_{f}(A)$ are Zariski-Samuel associated primes.

(ii) The primes in $\mathcal{X}_{A}$ are either maximal members in the set

$$
\{\sqrt{A: x}: x \in R \backslash A\}
$$

of radical ideals or unions of incomparable primes in $\operatorname{Ass}_{f}(A)$.

(iii) If $P=\sqrt{A: y}$ is maximal in the set (1), then $A: y$ is P-primary.

Proof. (i) By Heinzer and Ohm [14, Lemma 3.2], the members of $\operatorname{Ass}_{f}(A)$ are all Zariski-Samuel associated primes, if the radical ideals containing $A$ satisfy the ascending chain condition (cf. Lemma 1.5). Then $\operatorname{Ass}(A)$ is the set union of $\operatorname{Ass}_{f}(A)$ and unions of incomparable primes in $\operatorname{Ass}_{f}(A)$. (That the second kind of ideals may exist is illustrated by Example 4.5 infra.)

(ii) By Lemma 1.5(ii), the set $\{\sqrt{A: x}: x \in R \backslash A\}$ of radical ideals contains maximal members. Let $A: y(y \in R \backslash A)$ be an ideal with maximal radical $\sqrt{A: y}$. Lemma 1.5(iii) guarantees that $A: y$ has at most finitely many minimal primes; thus there are finitely many primes $P_{1}, \ldots, P_{m}$ with $\sqrt{A: y}=P_{1} \cap \cdots \cap P_{m}$, where no $P_{i}$ may be omitted. If $m>1$, pick a $u \in\left(P_{1} \cap \cdots \cap P_{m-1}\right) \backslash P_{m}$ and a $v \in P_{m} \backslash\left(P_{1} \cap \cdots \cap P_{m-1}\right)$. Then $u^{k} \notin A: y$ for any $k>0$, so by the maximal 
choice of $\sqrt{A: y}$, we must have $\sqrt{A: y u^{k}}=P_{1} \cap \cdots \cap P_{m}$ for every $k>0$. There is certainly a $k>0$ such that $(u v)^{k} \in A: y$. Hence we obtain $v^{k} \in A: y u^{k}$, so $v \in \sqrt{A: y u^{k}} \subseteq P_{1} \cap \cdots \cap P_{m}$, a contradiction. We have thus shown that the radical of $A: y$ is the prime ideal $P=P_{m}$.

(iii) To show that $A: y$ is $P$-primary, let $r, s \in R$ such that $r s \in A: y$, but $r \notin A: y$. Then by maximality again $\sqrt{A: r y}=P$, whence $s \in A: r y \subseteq P$.

Note that from Lemma 2.5 it follows that if $R$ is a Prüfer domain and $\operatorname{Spec}(R / A)$ is a Noetherian space, then every Krull associated prime of $A$ is a Zariski-Samuel associated prime of $A$.

Example 2.6. For an ideal $A$, the set $\mathcal{X}_{A}$ need not be finite even if $R$ has Noetherian spectrum. Let $F$ be a field, and $x, y$ indeterminates over $F$. Let $V$ be the discrete valuation domain obtained by localizing the polynomial $\operatorname{ring} F[x, y]$ at the prime ideal generated by $y$. Then $V=F(x)+P$, where $P$ denotes the maximal ideal of $V$.

It is easily seen that $R=F[x]+P$ is a Prüfer domain with $P$ as the unique prime ideal of height one. Evidently, the factor $\operatorname{ring} R / P$ is isomorphic to the polynomial ring $F[x]$, and as a result, $R$ has infinitely many maximal ideals of height 2 .

Consider the ideal $A=y R$. Manifestly, Spec $R$ is Noetherian. It is straightforward to check that each of the height 2 maximal ideals of $R$ consists of zero-divisors in $R / A$ and is a Krull associated prime to $A$. Hence $\mathcal{X}_{A}$ is an infinite set.

For an ideal $A$ of a Noetherian domain $R$, it may happen that there exist prime ideals $P \subset Q$ with $P$ and $Q$ in $\operatorname{Ass}(A)$ and yet there exist infinitely many prime ideals $L$ with $P \subset L \subset Q$ and $L \notin \operatorname{Ass}(A)$. Consider, for example, $R=F[x, y, z]$, where $x, y, z$ are indeterminates over a field $F$ and $A=\left(x^{2}, x y, x z\right) R$. Then $\operatorname{Ass}(A)=\{P, Q\}$, where $P=x R$ and $Q=(x, y, z) R$ and there exist infinitely many prime ideals $L$ such that $P \subset L \subset Q$ (see, for example [18, page 7]. Moreover, $P$ is the radical of $A$ and $Q$ is the adjoint of the primal ideal $A$.

To illustrate the properties of $\operatorname{Ass}(A)$ in a non-Noetherian setting, we turn our attention to arithmetical rings. In this case we can assert very specific properties of the set $\operatorname{Ass}(A)$. It is interesting to compare Proposition 2.7 to the example of the preceding paragraph.

Proposition 2.7. Let $R$ be an arithmetical ring and let $A$ be a proper ideal of $R$. If $P \in \operatorname{Ass}(A)$, then $Q \in \operatorname{Ass}(A)$ for every prime ideal $Q$ of $R$ such that $A \subseteq Q \subseteq P$.

Proof. By Lemma 2.4 it is enough to prove the proposition in the case where $R$ is a valuation ring. Furthermore, we may assume that $A=(0)$. If $P \in \operatorname{Ass}(A)$, then $R_{P}$ is a valuation ring whose maximal ideal consists of zero divisors. Thus if $Q \subseteq P$, then by Lemma 1.1 of [11], $Q R_{Q}$ consists of zero divisors of $R_{Q}$, and it follows that $Q \in \operatorname{Ass}(A)$.

We conclude from Proposition 2.7 that if $R$ is a valuation ring and $A \neq 0$ is a proper ideal of $R$, then $\operatorname{Ass}(A)$ is exactly the set of all primes between the radical of $A$ and the adjoint prime of $A$. In this case, all the primes in $\operatorname{Ass}(A)$ are Zariski-Samuel associated primes with the possible exception of those primes that are infinite unions of strictly smaller primes.

Let $A$ be a nonzero proper ideal of a Prüfer domain $R$ and let $M$ be a maximal ideal of $R$ with $A \subseteq M$. Since $R_{M}$ is a valuation domain, $\operatorname{End}\left(A R_{M}\right)=R_{P}$ for 
some $P \in \operatorname{Spec} R$ with $P \subseteq M$. Let

$$
\mathcal{E}_{A}=\left\{P \in \operatorname{Spec} R: R_{P}=\operatorname{End}\left(A R_{Q}\right) \text { for some prime ideal } Q \text { containing } A\right\} .
$$

Proposition 2.8. Let $A$ be a nonzero proper ideal of a Prüfer domain $R$. Then $\operatorname{Ass}(A)=\mathcal{E}_{A}$. In particular, if $P \in \mathcal{X}_{A}$, then there exists a maximal ideal $M$ of $R$ such that $\operatorname{End}\left(A_{M}\right)=R_{P}$.

Proof. We first show $\operatorname{Ass}(A) \subseteq \mathcal{E}_{A}$. By Proposition 2.7, it is enough to verify that $\mathcal{X}_{A} \subseteq \mathcal{E}_{A}$. Let $P \in \mathcal{X}_{A}$, and let $M$ be some maximal ideal of $R$ containing $P$. Since $P \in \operatorname{Ass}(A), P_{M} \in \operatorname{Ass}\left(A_{M}\right)$ by Lemma 2.4. If $Q_{M}$ is the adjoint prime of the irreducible (hence, by Lemma 1.2, primal) ideal $A_{M}$, then $P_{M} \subseteq Q_{M}$, and $Q_{M} \in \operatorname{Ass}\left(A_{M}\right)$ by Corollary 2.3. Thus, by Lemma 2.4, $Q \in \operatorname{Ass}(A)$. Since $P \subseteq Q$ and $P$ is in $\mathcal{X}_{A}$, it must be the case that $P=Q$. Thus $A_{M}$ has adjoint prime $P_{M}$, and by Lemma 2.4 in [7, $\operatorname{End}\left(A_{M}\right)=R_{P}$. Consequently, $P \in \mathcal{E}_{A}$.

To prove the converse, by Proposition 2.7, it suffices to show that every maximal element $P$ (with respect to set inclusion) of $\mathcal{E}_{A}$ is a member of $\operatorname{Ass}(A)$. Note that every member of $\mathcal{E}_{A}$ is contained in at least one maximal element of $\mathcal{E}_{A}$ and that such a maximal element $P$ must satisfy $\operatorname{End}\left(A_{M}\right)=R_{P}$ for some maximal ideal $M$ of $R$. Let $M$ be such a maximal ideal of $R$. By Lemma 2.4 in [7], $A_{M}$ is a primal ideal with adjoint prime $P_{M}$. It follows that $P_{M} \in \operatorname{Ass}\left(A_{M}\right)$; hence $P \in \operatorname{Ass}(A)$ by Lemma 2.4

The following example shows that in the setting of Prüfer domains, even with additional hypothesis such as Noetherian spectrum, the maximal prime divisors of an ideal need not be Krull associated primes. (Compare this to the Noetherian case, in which maximal prime divisors are always associated primes.) In [6], we consider this problem in more depth.

Example 2.9. There exists a 2-dimensional Prüfer domain $R$ for which $\operatorname{Spec}(R)$ is Noetherian and for which there exist an ideal $A$ and a finitely generated maximal ideal $M$ such that $M$ is a maximal prime divisor of $A$ and yet $A: M=A$ and $M \notin \operatorname{Ass}(A)$. Let $D$ be a Dedekind domain with non-torsion class group and let $P$ be a maximal ideal of $D$ that is not the radical of a principal ideal. Let $x$ be an indeterminate over the field of fractions $K$ of $D$ and let $V=K[x]_{(x K[x])}=K+Q$, where $Q$ is the maximal ideal of $V$. Define $R=D+Q$. Then $R$ is a 2-dimensional Prüfer domain with $Q$ as the unique prime ideal of $R$ of height one. The maximal ideals of $R$ are all of height two and have the form $M^{\prime}=P^{\prime} R$, where $P^{\prime}$ is a maximal ideal of $D$. Thus Spec $R$ is Noetherian. Let $E=\bigcup_{n=1}^{\infty} D:_{K} P^{n}$ be the $P$-transform of $D$. Consider the ideal $A=x E R$ of $R$ and the maximal ideal $M=P R$ of $R$. Since $E D_{P}=K$, it follows that $A R_{M}=Q R_{M}$ and $M \notin \operatorname{Ass}(A)$. However, for $P^{\prime}$ a maximal ideal of $D$ with $P^{\prime} \neq P$ and $M^{\prime}=P^{\prime} R$, we have $A R_{M^{\prime}}=x R_{M^{\prime}}$. Therefore $M^{\prime} \in \operatorname{Ass}(A)$. Since every element of $P$ is contained in a maximal ideal of $D$ distinct from $P$, it follows that every element of $M$ is non-prime to $A$ and $M$ is a maximal prime divisor of $A$.

\section{ISOLATED COMPONENTS AND PRIMAL IDEALS}

In view of Lemma 1.2, every ideal of a ring is the intersection of primal ideals. Also, every ideal is the intersection of its isolated components. We would like to have an intersection representation of an ideal with components that are both 
isolated components and primal ideals with distinct adjoint primes; in this way we will have more information about the components and the decomposition.

In the representation of an ideal as the intersection of its isolated components we do not need all of them to obtain the ideal: those belonging to maximal prime divisors, the principal components (Hauptkomponenten) in the sense of Krull [20. page 16], would perfectly suffice (see the comment after Lemma 3.1). However, an objection to using these components is that they need not be ideals with wellunderstood properties; for example, they may fail to be primal. Therefore, we focus our attention on the isolated components belonging to the maximal Krull associated primes; they will provide sufficiently many isolated components to represent the ideals, as is shown by the next result.

Lemma 3.1. For every proper ideal $A$ of the ring $R$ we have

$$
A=\bigcap_{P \in \mathcal{X}_{A}} A_{(P)} \text {. }
$$

Proof. Suppose $x \notin A$. If $Q$ is a minimal prime of $A: x$, then $Q$ is contained in some $P \in \mathcal{X}_{A}$. Hence $A: x \subseteq P$, and it follows that $x \notin A_{(P)}$. Consequently, $x \notin \bigcap_{P \in \mathcal{X}_{A}} A_{(P)}$, and we conclude that $A=\bigcap_{P \in \mathcal{X}_{A}} A_{(P)}$.

Observe that every prime $P$ in $\mathcal{X}_{A}$ is contained in a maximal prime divisor $P^{\prime}$ of $A$. The principal component of $A$ with respect to $P^{\prime}$ is obviously contained in the isolated component of $A$ with respect to $P$. Therefore, the decomposition of Lemma 3.1 continues to hold if the intersection is taken not over the primes in $\mathcal{X}_{A}$ but over the maximal prime divisors of $A$. In particular, the following result was proved by Krull [20, page 16]:

Corollary 3.2. Every ideal $A$ is the intersection of its principal components $A_{(P)}$, $P$ a maximal prime divisor of $A$. In particular, if $A$ has only finitely many maximal prime divisors, $P_{1}, \ldots, P_{n}$, then $A$ is the intersection of finitely many principal components $A_{\left(P_{i}\right)}$.

It is natural to wonder when the isolated components, and in particular those in the intersection presented in Lemma 3.1, are primal ideals. Examples given by Nagata [24] and by Gilmer [8] show that if $P$ is a maximal prime divisor of the ideal $A$, then the ideal $A_{(P)}$ need not be $P$-primal. In these examples, $A$ has infinitely many maximal prime divisors. In Example 3.8 below, we present an example where the ideal $A$ has precisely two maximal prime divisors and where the principal component with respect to each of these maximal prime divisors is not a primal ideal.

There are important special cases in which isolated components are always primal, viz. the arithmetical rings.

Lemma 3.3. If $A$ is an ideal of an arithmetical ring, then $A_{(P)}$ is an irreducible (hence primal) ideal for every prime ideal $P$ containing $A$. Its adjoint prime is contained in $P$.

Proof. By Remark 1.6, $A_{P}$ is irreducible if and only if $A_{(P)}$ is irreducible. Since $R_{P}$ is a valuation ring, it follows that $A_{(P)}$ is an irreducible ideal of $R$. By Lemma 1.3 , the adjoint prime of $A_{(P)}$ is contained in $P$.

Let $P$ be a prime ideal containing the ideal $A$. The isolated $P$-component $A_{(P)}$ is a $P$-primary ideal if and only if $P$ is a minimal prime of $A$. The most important 
criterion for the primality of the isolated components is given by the equivalence of (1) and (3) of [21, Proposition 1.1], as we record in Theorem [3.4. Since our definition of Krull associated prime is different from that given in [21], we give a short direct proof of the result.

Theorem 3.4. Let $P$ be a prime ideal containing the ideal $A$. The isolated $P$ component $A_{(P)}$ of $A$ is P-primal if and only if $P$ is a Krull associated prime of $A$.

Proof. Since the elements of $R \backslash P$ are prime to $A_{(P)}, A_{(P)}$ is $P$-primal if and only if the elements of $P$ are nonprime to $A_{(P)}$. Equivalently, for every $x \in P$, there is a $z \notin A_{(P)}$ such that $x z \in A_{(P)}$, i.e., $x z u \in A$ for some $u \in R \backslash P$. Thus $x \in A: z u$, where $z u \notin A_{(P)}$. The last relation says that $A: z u \subseteq P$, so $P$ is a Krull associated prime of $A$.

We summarize our findings in

Theorem 3.5. Every proper ideal $A$ of a ring $R$ can be written as an intersection

$$
A=\bigcap_{P \in \mathcal{X}_{A}} A_{(P)},
$$

where the (isolated) components $A_{(P)}$ are primal ideals with distinct, incomparable adjoint primes $P$.

We call the decomposition of Theorem 3.5 the canonical primal decomposition of $A$. Example 2.6 shows that the canonical primal decomposition may be infinite even if Spec $R$ is Noetherian.

For a maximal prime divisor $P$ of an ideal $A$, the question was raised in 5 , page $3]$ whether or not the principal $P$-component $A_{(P)}$ is equal to the intersection of all primal ideals that contain $A$ and have their associated adjoint prime contained in $P$. It follows from Theorem 3.4 and observations made in [5] that the answer to this question is affirmative if $P \in \operatorname{Ass}(A)$. We show in Corollary 3.6 that the answer is affirmative in general.

Corollary 3.6. Let $P$ be a maximal prime divisor of an ideal $A$ of the ring $R$. Let $A_{(P)}$ denote the principal $P$-component of $A$ and let $A_{(P)}^{*}$ be the intersection of all primal ideals that contain $A$ and have their associated adjoint prime contained in $P$. Then $A_{(P)}=A_{(P)}^{*}$.

Proof. It is shown in [5, page 3] that $A_{(P)} \subseteq A_{(P)}^{*}$, and it is easily seen that the elements of $R \backslash P$ are prime to $A_{(P)}$. Hence if $Q \in \operatorname{Ass}\left(A_{(P)}\right)$, then $Q \subseteq P$. Therefore the reverse inclusion follows from the canonical primal decomposition of $A_{(P)}$ given by Theorem 3.5. Indeed, if $Q \in \operatorname{Ass}\left(A_{(P)}\right)$, then by Lemma $2.4 Q \in \operatorname{Ass}\left(A_{P}\right)$, so that (again by Lemma 2.4) $Q \in \operatorname{Ass}(A)$. Thus by Theorem 3.5

$$
A_{(P)}=\bigcap\left\{A_{(Q)}: Q \in \operatorname{Ass}(A), Q \subseteq P\right\} .
$$

It is also true that $A_{(P)}$ is an intersection of $P$-primal ideals, as we show in Proposition 3.7 .

Proposition 3.7. Let $P$ be a prime ideal of the ring $R$. An ideal $A$ contained in $P$ is an intersection of $P$-primal ideals if and only if the elements of $R \backslash P$ are prime to $A$. In particular, $A_{(P)}$ is an intersection of $P$-primal ideals. 
Proof. If $A=\bigcap Q_{i}$, where each $Q_{i}$ is $P$-primal, then for $s \in R \backslash P$, we have $A: s=\left(\bigcap Q_{i}\right): s=\bigcap\left(Q_{i}: s\right)=\bigcap Q_{i}=A$. Thus the elements of $R \backslash P$ are prime to $A$.

To prove the converse, we may pass from $R$ to $R / A$ and assume that $A=(0)$. Then the elements of $R \backslash P$ are prime to (0), and $R$ is a subring of the localization $T=R_{P}$. We observe that if $J$ is a $P T$-primal ideal of $T$, then $I=J \cap R$ is $P$-primal in $R$. Indeed, $T$ being a localization of $R$ implies every ideal of $T$ is the extension of an ideal of $R$, so $I T=J$. Moreover for $r \in R$, we have $\left(I:_{R} r\right) T=I T:_{T} r$. It follows that $I$ is $P$-primal in $R$. Therefore it suffices to show that if $R$ is quasilocal with maximal ideal $P$, then $(0)$ is an intersection of $P$-primal ideals of $R$. Let $a$ be a nonzero element of $P$ and let $Q=a P$. Then $a \notin Q$ and $P=Q: a$, so $Q$ is $P$-primal. Thus (0) is an intersection of $P$-primal ideals.

Example 3.8. There exists a ring $R$ having Noetherian prime spectrum and precisely two maximal ideals, each of which is a maximal prime divisor of (0) but not a Krull associated prime of (0). Consider the polynomial ring $E=F[x, y, z]$ in three indeterminates over a field $F$ and the maximal ideals $N_{1}=(x, y, z) E$ and $N_{2}=(x, y, z-1) E$ of $E$. Let $D$ denote the localization of $E$ at the multiplicative set $E \backslash\left(N_{1} \cup N_{2}\right)$. Then $D$ is a unique factorization domain with precisely two maximal ideals, $N_{1} D$ and $N_{2} D$. Consider the height-two prime ideals $P_{1}=(x, z) D$ and $P_{2}=(y, z-1) D$, and let $C$ denote the $D$-module $C=\bigoplus D / p$, where $p$ varies over the height-one primes of $D$ other than $x D$ and $y D$. Let $B=C \oplus D / P_{1} \oplus D / P_{2}$.

Define $R$ to be the direct sum of $D$ and $B$ made into a ring via Nagata's idealization [25] (see Exercises 6,7 on pp. 62-63 in Kaplansky [18). It is clear that $\operatorname{Spec} R$ is isomorphic to $\operatorname{Spec} D$. Thus $\operatorname{Spec} R$ is Noetherian. Since $D$ is an integral domain, it follows that $B$ is the nilradical of $R$ and $R$ has precisely two maximal ideals, $M_{1}=N_{1} D \oplus B$ and $M_{2}=N_{2} D \oplus B$. Moreover, every element of $M_{1} \cup M_{2}$ is a zero divisor, so $R$ is equal to its total quotient ring. For $i=1,2$, let $A_{i}=\left\{r \in R\right.$ : there exists $s \in R \backslash M_{i}$ such that $\left.r s=0\right\}$ denote the principal component of the zero ideal of $R$ with respect to the maximal ideal $M_{i}$. Then $A_{1}$ and $A_{2}$ are contained in $B ; A_{1}$ is the direct sum of $D / P_{2}$ with $\bigoplus D / p$, where $p$ varies over the height-one primes of $D$ not contained in $M_{1}$ and $A_{2}$ is the direct sum of $D / P_{1}$ with $\bigoplus D / p$, where $p$ varies over the height-one primes of $D$ not contained

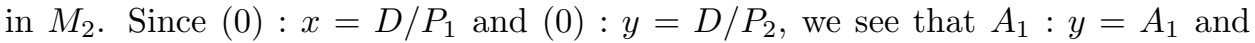
$A_{2}: x=A_{2}$. Therefore the ideal $A_{i}$ is not $M_{i}$-primal and $M_{i}$ is not an associated prime of (0). Indeed, the ideals $A_{1}$ and $A_{2}$ are not even primal, since the set of elements non-prime to $A_{i}$ does not form an ideal. For example, the elements $y+z$ and $z$ are non-prime to $A_{1}$, while their difference $y$ is prime to $A_{1}$.

\section{IRREDUNDANT PRIMAL DECOMPOSITIONS}

We wish to consider primal decompositions of an ideal $A$ with distinct adjoint primes, and to examine when such decompositions are irredundant and unique in some sense. Recall that if an ideal $A$ is represented as an intersection of ideals $A=\bigcap_{j} B_{j}$, then a component $B_{j}$ is said to be relevant in this representation if omitting $B_{j}$ increases the intersection. If each component $B_{j}$ is relevant, then the intersection is said to be irredundant.

In this section we concentrate on the general case and on rings with Noetherian spectra, while in the next section we deal with arithmetical rings. 
First, we verify the following lemma.

Lemma 4.1. Suppose that $A$ is a proper ideal of $R$, and $P \in \mathcal{X}_{A}$ has one of the following properties:

(i) $P$ is not contained in the set union of the primes $Q \in \mathcal{X}_{A}$ with $Q \neq P$.

(ii) $P$ is a Zariski-Samuel associated prime.

Then in the canonical primal decomposition (2) the isolated P-component $A_{(P)}$ is relevant.

Proof. (i) Suppose that $P$ is not contained in the set union of the primes $Q \in \mathcal{X}_{A}$ with $Q \neq P$. Then there exists an element $p \in P$ that fails to belong to any of these $Q \in \mathcal{X}_{A}$. By Theorem 3.4, $A_{(Q)}$ is a $Q$-primal ideal; thus $A_{(Q)}: p=A_{(Q)}$ for every $Q \neq P$. From (2) we obtain

$$
A: p=\left(A_{(P)}: p\right) \cap \bigcap_{Q \in \mathcal{X}_{A}, Q \neq P} A_{(Q)} .
$$

In view of Lemma 2.2, $A: p$ properly contains $A$, whence it is clear that (a) $A_{(P)} \subset A_{(P)}: p$; and (b) in (2) we cannot replace $A_{(P)}$ by the larger $A_{(P)}: p$. Consequently, the component $A_{(P)}$ is relevant in (2).

(ii) Suppose that $P$ is a Zariski-Samuel associated prime to $A$. Then by definition there exists an element $y \notin A$ such that $P=\sqrt{A: y}$. If $P \neq Q \in \mathcal{X}_{A}$, then $A_{(Q)}: y \subseteq Q$ is impossible, as it would imply $P=\sqrt{A: y} \subseteq \sqrt{A_{(Q)}: y} \subseteq Q$. We conclude that $y \in A_{(Q)}$ holds for all $Q \neq P$. In other words, $y \in \bigcap_{Q \in \mathcal{X}_{A}, Q \neq P} A_{(Q)}$. But then $A_{(P)}: y=A: y \subseteq P$, which shows that $y \notin A_{(P)}$, establishing the claimed relevance of $A_{(P)}$.

In conclusion, from Theorem 3.5 and Lemma 4.1 we have established:

Theorem 4.2. Let $A$ be a proper ideal of the ring $R$. If each $P \in \mathcal{X}_{A}$ has the property that either (i) $P$ is not contained in the set union of the other primes in $\mathcal{X}_{A}$, or (ii) $P$ is a Zariski-Samuel associated prime of $A$, then the canonical primal decomposition $A=\bigcap_{P \in \mathcal{X}_{A}} A_{(P)}$ is irredundant.

If each $P \in \mathcal{X}_{A}$ is a Zariski-Samuel prime of $A$, we prove that a stronger form of irredundancy holds. An intersection $A=\bigcap_{j} B_{j}$ is said to be residually maximal at $B_{j}$ if the intersection no longer equals $A$ whenever $B_{j}$ is replaced by a residual $B_{j}: c$ that properly contains $B_{j}$. The intersection $A=\bigcap_{j} B_{j}$ is said to be residually maximal if it is residually maximal at each $B_{j}$. Residually maximal intersections are evidently irredundant.

Proposition 4.3. If $P \in \mathcal{X}_{A}$ is a Zariski-Samuel associated prime of $A$, then the canonical primal decomposition $A=\bigcap_{Q \in \mathcal{X}_{A}} A_{(Q)}$ is residually maximal at $A_{(P)}$. If each $P \in \mathcal{X}_{A}$ is a Zariski-Samuel associated prime of $A$, then the canonical primal decomposition is residually maximal.

Proof. Supposing that the component $A_{(P)}$ can be replaced by $A_{(P)}: c$ for some $c \in R$, we show that such a residual cannot be larger than $A_{(P)}$. We argue as in the proof of Lemma 4.1(ii) (keeping the same notation) that in this case

$$
A: y=A_{(P)}: y=\left(A_{(P)}: c\right): y=\left(A_{(P)}: y\right): c .
$$

Thus $c$ is prime to the $P$-primary ideal $A: y$ (see Lemma 2.5(iii)), i.e. $c \notin P$. Therefore, $A_{(P)}: c=A_{(P)}$, since by Theorem $3.4 A_{(P)}$ is $P$-primal. 
We now give several examples to illustrate our theorems.

Example 4.4. An irredundant primal decomposition of an ideal A of a Noetherian ring $R$ need not consist of isolated components. Consider the polynomial $\operatorname{ring} R=$ $F[x, y, z]$ in three indeterminates with coefficients in a field $F$. The ideal $A=$ $\left(x^{2} y, x y^{2} z^{2}\right) R$ has a primary decomposition

$$
A=x R \cap y R \cap\left(x^{2}, y^{2}\right) R \cap\left(x^{2}, z^{2}\right) R
$$

with radicals $x R, y R,(x, y) R,(x, z) R$, respectively. The set $\mathcal{X}_{A}$ consists of two prime ideals (they are Zariski-Samuel associated primes):

$$
\sqrt{A: x y z^{2}}=(x, y) R \text { and } \sqrt{A: x y^{2} z}=(x, z) R .
$$

The canonical primal decomposition of $A$ given by (2) of Theorem 3.5 is

$$
A=A_{(x, y) R} \cap A_{(x, z) R},
$$

where

$$
A_{(x, y) R}=\left(x^{2} y, x y^{2}\right) R \quad \text { and } \quad A_{(x, z) R}=\left(x^{2}, x z^{2}\right) R .
$$

Note that $A=\left(x^{2} y, x y^{2}\right) R \cap\left(x^{2}, z^{2}\right) R$ and $A=\left(x^{2} y, y^{2}\right) R \cap\left(x^{2}, x z^{2}\right) R$ are representations of $A$ as intersections of primal ideals; however, the primal ideals $\left(x^{2}, z^{2}\right) R$ and $\left(x^{2} y, y^{2}\right) R$ are not isolated components of $A$.

Example 4.5. There exists a ring $R$ with Noetherian prime spectrum such that $R$ has a P-primal ideal $A$ that can be represented as an irredundant intersection of primal isolated components whose associated adjoint primes are different from the adjoint prime $P$ of $A$. Consider the localized polynomial ring $D=F[x, y]_{(x, y) F[x, y]}$, where $x, y$ are two indeterminates over a field $F$. Visibly, $D$ is a 2-dimensional local UFD. Let $B$ be the $D$-module which is the direct sum of the $D / p D$ as $p$ varies over the non-associate irreducible elements of $D$.

As in Example 3.8, define $R$ to be the direct sum of $D$ and $B$ made into a ring via Nagata's idealization. It is clear that $\operatorname{Spec} R$ is isomorphic to $\operatorname{Spec} D$. Hence Spec $R$ is Noetherian. Let $M=(x, y) D+B$ denote the maximal ideal of $R$ and let $A=(0)$ in $R$. Since $M$ consists of zero-divisors, $A$ is a primal ideal of $R$ whose adjoint prime is $M$.

The set $\mathcal{X}_{A}$ consists of the single element $M$. Moreover, the height-one primes $P$ of $R$ have the form $P=p D+B$, where $p$ is an irreducible element of $D$. Hence $M$ is the union of the height one primes of $R$, each of which is a Zariski-Samuel associated prime of $A$. For $P=p D+B$ a height-one prime of $R$, the isolated component $A_{(P)}$ is the submodule of $B$ that is the direct sum of $D / q D$ as $q$ varies over the nonassociate irreducible elements of $D$ distinct from $p$. Thus the primal ideal $A$ of $R$ can be represented with primal components both as $A$ (one component), and also as an irredundant intersection $A=\bigcap A_{(P)}$ of $P$-primal ideals, where $P$ varies over the height-one primes of $R$ (infinitely many components).

As Examples 4.4 and 4.5 show, an ideal may have various decompositions into the intersection of primal ideals. For primes $P \in \operatorname{Ass}(A)$, the isolated $P$-component $A_{(P)}$ of an ideal $A$ is certainly the minimal $P$-primal ideal containing $A$, so the representation established in Lemma 3.1 is the minimal one in the obvious sense. Since there might exist other decompositions into primal components, the usual question arises: to what extent is the representation of an ideal as an intersection of primal isolated components unique? Needless to say, we cannot expect complete 
uniqueness, not even for irredundant representations with distinct adjoint primes, since this fails already in the Noetherian case. However, the uniqueness of the set of adjoint primes can be ascertained if we assume some sort of maximality (in the Noetherian case, it was assumed that the intersection was reduced; see [5]).

By a primal isolated component of an ideal $A$ we mean a primal ideal $B$ such that $B=A_{(Q)}$ for some prime ideal $Q$. If $P$ is the adjoint prime of $B$, then necessarily $P \subseteq Q$. It is possible that $P$ is properly contained in $Q$. However, regardless of whether this containment is proper, it follows that $A_{(P)}=A_{(Q)}$. For $P \subseteq Q$ implies $A_{(Q)} \subseteq A_{(P)}$, and by Lemma $1.3(\mathrm{i}), B=B_{(P)}$. Since $A \subseteq B$, we have $B=A_{(Q)} \subseteq A_{(P)} \subseteq B$. Thus if $B$ is a primal isolated component of $A$, then $B=A_{(P)}$ for some $P \in \operatorname{Ass}(A)$. In Theorem 4.6 we pinpoint a necessary and sufficient condition for $P \in \mathcal{X}_{A}$ to appear in every representation of $A$ as an intersection of a subset of its primal isolated components.

Theorem 4.6. If $A$ is an ideal of the ring $R$ and $P \in \mathcal{X}_{A}$, then the following statements are equivalent for $P$ :

(i) $P$ is a Zariski-Samuel associated prime of $A$.

(ii) The isolated component $A_{(P)}$ must appear in every representation of $A$ as an intersection of primal isolated components of $A$.

Proof. (i) $\Rightarrow$ (ii) The proof of Lemma 4.1(ii) applies, since it requires only that $Q \in \operatorname{Ass}(A)$.

(ii) $\Rightarrow$ (i) Suppose that $P$ is not a Zariski-Samuel associated prime of $A$. We claim that $A=\bigcap_{Q \neq P} A_{(Q)}$, where $Q$ ranges over the members of $\operatorname{Ass}(A)$ distinct from $P$, thus contradicting (ii). To prove this, it suffices to show that for every $y \in R \backslash A$, there exists $Q \in \operatorname{Ass}(A)$ with $Q \neq P$ and $A: y \subseteq Q$. Let $y \in R \backslash A$. By assumption $\sqrt{A: y} \neq P$, so there exists a minimal prime $Q$ of $A: y$ with $Q \neq P$. Necessarily, $Q \in \operatorname{Ass}(A)$.

Corollary 4.7. For an ideal $A$ of the ring $R$ the following statements are equivalent.

(i) For each set of prime ideals $\mathcal{C} \subseteq \operatorname{Ass}(A)$ such that $A=\bigcap_{P \in \mathcal{C}} A_{(P)}$, it is the case that $\mathcal{X}_{A} \subseteq \mathcal{C}$.

(ii) Each $P \in \mathcal{X}_{A}$ is a Zariski-Samuel associated prime of $A$.

In particular, if each $P \in \mathcal{X}_{A}$ is a Zariski-Samuel associated prime of $A$, then the canonical primal decomposition $A=\bigcap_{P \in \mathcal{X}_{A}} A_{(P)}$ is irredundant.

Proof. That (i) implies (ii) follows from Theorems 3.4 and 4.6. To see that (ii) implies (i), suppose $A=\bigcap_{P \in \mathcal{C}} A_{(P)}$ for some $\mathcal{C} \subseteq \operatorname{Ass}(A)$. By Theorem 4.6, for each $P \in \mathcal{X}_{A}$, we have $A_{(P)}=A_{(Q)}$, for some $Q \in \mathcal{C}$. Since $P, Q \in \operatorname{Ass}(A)$, we have by Theorem 3.4 that $A_{(P)}$ is $P$-primal and $A_{(Q)}$ is $Q$-primal. Since $A_{(P)}=A_{(Q)}$, we conclude that $P=Q$.

Remark 4.8. If $P \in \mathcal{X}_{A}$ is of the form $P=\sqrt{A: x}$, then $A: x$ is $P$-primary. For if $r, s \in R$ are such that $r s \in A: x$ and $r \notin A: x$, then $A: r x$ is a proper ideal and $P \in \mathcal{X}_{A}$ implies that $P=\sqrt{A: r x}$. Hence $s \in A: r x \subseteq P$, so $A: x$ is $P$-primary.

Example 4.5 shows that the canonical primal decomposition of $A$ given in (2) of Theorem 3.5 may be irredundant, but not the unique representation of $A$ as an irredundant intersection of pairwise incomparable isolated primal components of $A$. For the ideal $A=y R$ of Example [2.6, the canonical primal decomposition of $A$ given in (2) of Theorem 3.5 is irredundant, and yet the set $\mathcal{X}_{A}$ is infinite. 
There are also examples where $A$ is an ideal of a one-dimensional Prüfer domain and the canonical primal decomposition given in (2) of Theorem 3.5 is redundant, but a proper subset of $\mathcal{X}_{A}$ gives an irredundant representation of $A$. Indeed, it can happen that all but one of the elements of $\mathcal{X}_{A}$ is a Zariski-Samuel prime of $A$ and that $A$ is the intersection of its primal components with respect to this proper subset of $\mathcal{X}_{A}$ (cf. Example 2.2 in [14]).

We would like to have conditions that are both necessary and sufficient for irredundance of the canonical primal decomposition $A=\bigcap_{P \in \mathcal{X}_{A}} A_{(P)}$.

The following interesting example, shown to us by Alan Loper and included here with his permission, shows that the sufficient conditions of Theorem 4.2 are not in general necessary conditions for irredundance. The example is a Prüfer domain $R$ having the following property:

(\#) the representation $R=\bigcap\left\{R_{P}: P \in \operatorname{Max}(R)\right\}$ is irredundant.

The integral domain $R$ is constructed so that it has a nonzero principal ideal $A=y R$ that is contained in every maximal ideal of $R$. Since $R$ is Prüfer and $A$ is principal, it follows from Proposition 2.8 that $\mathcal{X}_{A}=\operatorname{Max}(R)$. Moreover, the representation $A=\bigcap_{P \in \mathcal{X}_{A}} A_{(P)}$ is irredundant, and yet there exists $P \in \mathcal{X}_{A}$ such that $P$ is contained in the union of the prime ideals of $\mathcal{X}_{A}$ distinct from $P$ and $P$ is not a Zariski-Samuel associated prime of $A$.

Example 4.9 (Alan Loper). Let $V$ be a valuation domain on a field $K$ such that the maximal ideal $M$ of $V$ is the union of the prime ideals properly contained in $M$. Let $E=\left\{a_{1}, a_{2}, a_{3}, a_{4}\right\}$, where $\left\{a_{i}\right\}_{i=1}^{4}$ are nonzero elements of $M$ having the property that

$$
a_{4} V=a_{3} V \supset\left(a_{3}-a_{4}\right) V \supset a_{2} V \supseteq a_{1} V .
$$

Thus if $v$ is a valuation on $K$ associated to the valuation domain $V$, then

$$
v\left(a_{4}\right)=v\left(a_{3}\right)<v\left(a_{3}-a_{4}\right)<v\left(a_{2}\right) \leq v\left(a_{1}\right) .
$$

Let $x$ be an indeterminate and let $D=\operatorname{Int}(E, V)=\{f(x) \in K[x]: f(E) \subseteq V\}$ be the ring of integer-valued polynomials on the set $E$. It is well-known that $D$ is a Prüfer domain [22, Corollary 7]. Moreover, $D$ has precisely four maximal ideals of infinite height. These are the maximal ideals containing $M D$. They are $M_{i}$, $1 \leq i \leq 4$, where $M_{i}=\left\{f(x) \in K[x]: f\left(a_{i}\right) \in M\right\}$. The other maximal ideals of $D$ are of height one and are the contraction to $D$ of maximal ideals of $K[x]$. Each of the maximal ideals of $K[x]$ other than $\left(x-a_{i}\right) K[x]$ intersects $D$ in a maximal ideal of $D$, while $\left(x-a_{i}\right) K[x] \cap D$, for $1 \leq i \leq 4$, is a height-one prime of $D$ that is contained in $M_{i}$. Each of the valuation domains $D_{M_{i}}$ has the property that its maximal ideal is the union of the prime ideals it properly contains. Therefore $M_{i}$ in $D$ is the union of the prime ideals it properly contains.

The following argument shows that $M_{i}$, for $1 \leq i \leq 4$, is contained in the union of the other maximal ideals of $D$. We have $D \subseteq K[x]$ and $M_{i} \cap K=M$ for each $i$ with $1 \leq i \leq 4$. Therefore every element of $K$ that is in one of the $M_{i}$ is in all four of the $M_{i}$. A nonconstant polynomial $f(x) \in D \subseteq K[x]$ that is in one of that $M_{i}$ and is not of the form $f(x)=d\left(x-a_{j}\right)^{n}$, where $d \in K, n$ is a positive integer and $1 \leq j \leq 4$, is easily seen to be also in another maximal ideal of $D$. Thus, assume $f(x)=d\left(x-a_{j}\right)^{n} \in D$. By definition of $D, f\left(a_{i}\right) \in V$ for $1 \leq i \leq 4$. Also $f\left(a_{j}\right)=0 \in M$, so $f(x) \in M_{j}$. Using the fact that

$$
v\left(a_{4}\right)=v\left(a_{3}\right)<v\left(a_{3}-a_{4}\right)<v\left(a_{2}\right) \leq v\left(a_{1}\right),
$$


it follows by examining cases that at most two of the values $v\left(f\left(a_{i}\right)\right)$ can be 0 . Therefore $f(x)$ is in at least two of the $M_{j}$.

Let $y$ be an indeterminate over $K(x)$, let $Q=y K(x)[y]_{(y)}$ and let $R=D+Q$. For each maximal ideal $P$ of $R$, we have $P=(P \cap D) R$ and $P \cap D$ is a maximal ideal of $D$. It follows that $M_{i} R$, for $1 \leq i \leq 4$, is a maximal ideal of $R$ that is contained in the union of the other maximal ideals of $R$.

To show that $R$ satisfies property (\#), it suffices to show that for each maximal ideal $P$ of $R$ there exists a finitely generated ideal contained in $P$ but not contained in any other maximal ideal of $R$. Since $Q$ is a height-one prime ideal of $R$ that is contained in every nonzero prime ideal of $R$ and since $D=R / Q$, it suffices to show this property for $D$. Each maximal ideal of $D$ is either the contraction of a maximal ideal of $K[x]$ or else in the set $\left\{M_{i}\right\}_{i=1}^{4}$. For each of the maximal ideals $P$ contracted from $K[x]$, there exists an element $f$, contained in $P$ and contained in no other maximal ideal of $D$, that is contracted from $K[x]$. By choosing an element of $P \backslash \bigcup_{i=1}^{4} M_{i}$, we see that $P$ is the radical of a finitely generated ideal of $D$. Thus each of the maximal ideals of $D$ contracted from $K[x]$ is the radical of a finitely generated ideal. Since a nonzero element of $D$ is contained in only finitely many maximal ideals of $K[x]$, we also easily get, for $1 \leq i \leq 4$, the existence of a finitely generated ideal $A_{i}$ contained in $M_{i}$ such that $A_{i}$ is contained in no other maximal ideal of $D$. Therefore $D$ and $R=D+Q$ satisfy property (\#).

Let $A=y R$. Then $A$ is a nonzero principal ideal of $R$ that is contained in each maximal ideal of $R$. Hence by Proposition 2.8, $\mathcal{X}_{A}=\operatorname{Max}(R)$. Since $R$ satisfies property (\#), the representation of the principal ideal $A$ as $A=\bigcap_{P \in \mathcal{X}_{A}} A_{(P)}$ is irredundant, but $M_{i} R$, for $1 \leq i \leq 4$, is not a Zariski-Samuel associated prime of $A$, and $M_{i} R$ is contained in the union of the prime ideals of $\mathcal{X}_{A}$ distinct from $M_{i} R$. This completes the presentation of Example 4.9.

\section{IRREDUNDANT IRREDUCIBLE DECOMPOSITIONS}

In this section our focus is on irredundant decompositions of ideals $A$ into irreducible isolated components $A_{(P)}$. We prove a uniqueness statement about such decompositions, and then characterize those rings for which the canonical primal decomposition of every proper ideal $A$ is an irredundant representation of $A$ as an intersection of irreducible isolated components. In the statement of Theorem 5.1 we use the concept of a residually maximal intersection as defined in Section 4.

Theorem 5.1. Let $A$ be a proper ideal of a ring $R$. If $\mathcal{C}$ is a collection of prime ideals of $R$ and $A=\bigcap_{P \in \mathcal{C}} A_{(P)}$ is an irredundant intersection of irreducible isolated components of $A$, then this intersection is residually maximal and is the unique irredundant decomposition of $A$ into irreducible isolated components of $A$.

Proof. Since the isolated components $A_{(P)}$ are irreducible, they are primal. Without loss of generality, we assume $P$ is the adjoint prime of $A_{(P)}$ and $\mathcal{C}$ consists of the adjoint primes $P$ of the $A_{(P)}$. Thus $\mathcal{C} \subseteq \operatorname{Ass}(A)$, and $A=\bigcap_{P \in \mathcal{C}} A_{(P)}$ is an irredundant decomposition of $A$, where each $P \in \mathcal{C}$ is the adjoint prime of the irreducible ideal $A_{(P)}$.

If $P$ is a prime ideal of $R$ and $A=B \cap C$ for ideals $B$ and $C$ of $R$, then $A_{(P)}=B_{(P)} \cap C_{(P)}$. We will use this fact frequently in the proof.

Suppose that for some $P \in \mathcal{C}$ there exists an $x \in R$ such that

$$
A=\left(A_{(P)}: x\right) \cap\left(\bigcap\left\{A_{(Q)}: Q \in \mathcal{C}, Q \neq P\right\} .\right.
$$


Let $C=\bigcap_{Q \neq P} A_{(Q)}$. By Lemma 1.1, we have

$$
A_{(P)}=\left(A_{(P)}: x\right) \cap C_{(P)} .
$$

Since $A_{(P)}$ is irreducible, we have either $A_{(P)}=A_{(P)}: x$ or $A_{(P)}=C_{(P)}$. The latter equality, however, contradicts the irredundancy of the decomposition of $A$, so $A_{(P)}=A_{(P)}: x$. We conclude that any irredundant decomposition of $A$ into irreducible isolated components is residually maximal.

It remains to show that the representation $A=\bigcap_{P \in \mathcal{C}} A_{(P)}$ is unique among irredundant decompositions of $A$ into irreducible isolated components of $A$. Suppose $\mathcal{C}^{\prime}=\left\{Q_{i}\right\}_{i \in I} \subseteq \operatorname{Ass}(A)$ is such that $A=\bigcap_{i \in I} A_{\left(Q_{i}\right)}$ is also an irredundant decomposition of $A$ into irreducible components $B_{i}=A_{\left(Q_{i}\right)}$ of $A$ with adjoint primes $Q_{i}$. We show that for each $i^{\prime} \in I$, there exists $P_{i^{\prime}} \in \mathcal{C}$ such that $P_{i^{\prime}} \subseteq Q_{i^{\prime}}$. Fix $j \in I$, and define $C_{j}=\bigcap_{i \neq j} B_{i}$. We exhibit $P \in \mathcal{C}$ such that $P \subseteq Q_{j}$. Observe that for each $P \in \mathcal{C}$,

$$
A_{(P)}=\left(B_{j}\right)_{(P)} \cap\left(C_{j}\right)_{(P)} .
$$

Since $A_{(P)}$ is irreducible, it must be the case that $A_{(P)}=\left(B_{j}\right)_{(P)}$ or $A_{(P)}=\left(C_{j}\right)_{(P)}$. If the latter case holds for every $P \in \mathcal{C}$, then

$$
C_{j} \subseteq \bigcap_{P \in \mathcal{C}} A_{(P)}=A,
$$

and it follows that $\bigcap_{i \neq j} B_{i}=C_{j} \subseteq A$. However, this contradicts the assumption that the intersection $A=\bigcap_{i \in I} B_{i}$ is irredundant, so for some $P \in \mathcal{C}$ we must have $A_{(P)}=\left(B_{j}\right)_{(P)}$. Since $A_{(P)}$ is $P$-primal, so is $\left(B_{j}\right)_{(P)}$. However, by assumption, $B_{j}$ is $Q_{j}$-primal, so statement (iii) of Lemma 1.3 implies $P \subseteq Q_{j}$. We conclude that for each $i^{\prime} \in I$, there exists $P_{i^{\prime}} \in \mathcal{C}$ such that $P_{i^{\prime}} \subseteq Q_{i^{\prime}}$.

Applying a symmetrical argument to the decomposition $A=\bigcap_{i \in I} B_{i}$, we see that for each $P \in \mathcal{C}$, there exists $i \in I$ such that $Q_{i} \subseteq P$. Thus $P_{i} \subseteq Q_{i} \subseteq P$. Since the decomposition $A=\bigcap_{P \in \mathcal{C}} A_{(P)}$ is irredundant, it follows that $\mathcal{C}$ consists of incomparable primes. Thus $P_{i}=Q_{i}=P$, and $\mathcal{C} \subseteq\left\{Q_{i}: i \in I\right\}$. Also, the symmetry of our assumptions yields $\left\{Q_{i}: i \in I\right\} \subseteq \mathcal{C}$. We conclude that $\mathcal{C}=\left\{Q_{i}: i \in I\right\}$, and it follows that

$$
\left\{A_{(P)}: P \in \mathcal{C}\right\}=\left\{A_{\left(Q_{i}\right)}: i \in I\right\} .
$$

This proves the theorem.

Discussion 5.2. Assume that $\mathcal{C}$ is a subset of $\operatorname{Ass}(A)$ containing at least two elements. If $A=\bigcap_{P \in \mathcal{C}} A_{(P)}$ and if the intersection is irredundant, then $\mathcal{C}$ consists of incomparable primes. Also, for each $P \in \mathcal{C}$ there exists $Q \in \mathcal{X}_{A}$ such that $P \subseteq Q$, and it follows that $A_{(Q)} \subseteq A_{(P)}$. Thus if $\mathcal{C}^{\prime}=\mathcal{C} \backslash\{P\}$ and $B=\bigcap_{P^{\prime} \in \mathcal{C}^{\prime}} A_{\left(P^{\prime}\right)}$, then $A_{(Q)}$ is relevant in the intersection $A=A_{(Q)} \cap B$. Since $A_{(P)}$ is $P$-primal and $A_{(Q)}$ is $Q$-primal, we have $A_{(P)}=A_{(Q)}$ if and only if $P=Q$. If $A_{(Q)}$ is irreducible, we observe that $P=Q$. For $A=A_{(P)} \cap B$ implies $A_{(Q)}=A_{(P)} \cap B_{(Q)}$, and $P \neq Q$ and $A_{(Q)}$ irreducible imply $A_{(Q)}=B_{(Q)}$. But this implies $B \subseteq A_{(P)}$, which contradicts the irredundance of the intersection $A=A_{(P)} \cap B$. Therefore, if $A_{(Q)}$ is irreducible for each $Q \in \mathcal{X}_{A}$, then $\mathcal{C} \subseteq \mathcal{X}_{A}$.

From Discussion 5.2, we deduce the following corollary to Theorem 5.1.

Corollary 5.3. Let $A$ be an ideal of a ring $R$, and suppose $\mathcal{C} \subseteq \operatorname{Ass}(A)$. If $A_{(Q)}$ is irreducible for each $Q \in \mathcal{X}_{A}$ and $A=\bigcap_{P \in \mathcal{C}} A_{(P)}$ is an irredundant intersection, 
then $\mathcal{C} \subseteq \mathcal{X}_{A}$, and this is the unique decomposition of $A$ into an intersection of irreducible isolated components of $A$.

The irreducibility and isolation of the components in Theorem 5.1 are crucial to the assertion of uniqueness in the theorem. For $A$ as in Example 4.5 we have two distinct irredundant representations of $A$ as an intersection of primal isolated component ideals of $A$. Example 5.4 exhibits a situation where an ideal has two distinct irredundant representations as an intersection of primal isolated components, one of which consists of irreducible isolated components. It is interesting to contrast Theorem 5.1 with the situation for infinite irredundant primary decompositions of ideals in general. In this case, neither the primary ideals in the representation nor even the primes associated to these ideals need be unique, as is shown for example by Underwood in [31, Example 4.18]. On the other hand, it is readily seen that in a Noetherian ring there cannot exist an infinite irredundant intersection of ideals.

Example 5.4. An ideal may have two distinct decompositions as an irredundant intersection of primal isolated components, one of which consists of irreducible isolated components. Let $\left\{x_{n}: n \in \mathbb{N}\right\}$ be indeterminates over a field $F$ and let $R$ be the localization of the polynomial ring $F\left[x_{1}, x_{2}, \ldots\right]$ at the maximal ideal generated by $\left\{x_{n}: n \in \mathbb{N}\right\}$. Consider the ideal $A$ of $R$ generated by $\left\{x_{i} x_{j}: i \neq j\right\}$. Then, if $M$ is the unique maximal ideal of $R, R / A$ is one-dimensional with a unique maximal ideal $M / A$ and infinitely many minimal primes $P_{n} / A, n \in \mathbb{N}$, where $P_{n}=\left(\left\{x_{i}: i \in \mathbb{N}, i \neq n\right\}\right) R$. Moreover, $x_{n}$ is not in $P_{n}$ but is in every prime of $R$ containing $A$ other than $P_{n}$. The ideal $A$ of $R$ is $M$-primal and is the irredundant intersection of irreducible isolated components $A=\bigcap_{P \in \mathcal{C}} A_{(P)}$, where $\mathcal{C}=\left\{P_{n}: n \in \mathbb{N}\right\} \subset \operatorname{Ass}(A)$ consists of precisely the elements in $\operatorname{Ass}(A)$ other than $M$. Thus for this example, the canonical primal decomposition of $A$ given in (2) of Theorem 3.5 is trivial (that is, $A=A_{(M)}$ ) and yet there exists a representation $A=\bigcap_{P \in \mathcal{C}} A_{(P)}$ that is irredundant and consists of irreducible isolated components of $A$ and that is, by Theorem 5.1, unique among irredundant intersections of irreducible isolated components of $A$. However, the representation $A=\bigcap_{P \in \mathcal{C}} A_{(P)}$ is not unique among irredundant intersections of primal isolated components of $A$.

In light of Theorem 5.1, it is natural to consider rings $R$ for which every ideal $A$ is locally irreducible in the sense that its extension to $R_{M}$ is irreducible for each maximal ideal $M$ containing $A$. As noted in Section 1, these are precisely the arithmetical rings.

Returning to Theorem 5.1, we obtain a stronger result for arithmetical rings because of the following lemma, which asserts that irredundant primal decompositions in arithmetical rings must involve isolated components.

Lemma 5.5. Let $R$ be an arithmetical ring, and let $A$ be an ideal of $R$. Suppose $A=\bigcap_{i \in I} A_{i}$ is an intersection of ideals $A_{i}$ of $R$. For each $i \in I$, if $A_{i}$ is a primal ideal of $R$ that is relevant to this decomposition, then $A_{i}=A_{\left(P_{i}\right)}$, where $P_{i}$ is the adjoint prime of $A_{i}$.

Proof. Let $i \in I$, and suppose $A_{i}$ is relevant to the given decomposition. If $P_{i}$ is the adjoint prime of $A_{i}$, then $A_{i}=\left(A_{i}\right)_{\left(P_{i}\right)}$ in view of Lemma 1.3. Also,

$$
A_{\left(P_{i}\right)}=A_{i} \cap\left(\bigcap_{j \neq i} A_{j}\right)_{\left(P_{i}\right)} .
$$


By Remark 1.6, $A_{\left(P_{i}\right)}$ is an irreducible ideal of $R$. Thus if $A_{\left(P_{i}\right)} \neq A_{i}$, then $A_{\left(P_{i}\right)}=$ $\left(\bigcap_{j \neq i} A_{j}\right)_{\left(P_{i}\right)}$. But this implies that $\bigcap_{j \neq i} A_{j} \subseteq A_{i}$, contradicting the relevancy of $A_{i}$ to the decomposition. We conclude that $A_{\left(P_{i}\right)}=A_{i}$.

Corollary 5.6. Let $R$ be an arithmetical ring, and $A$ an ideal of $R$. If $A$ has an irredundant decomposition $A=\bigcap_{i \in I} A_{i}$ into irreducible ideals $A_{i}$, then this intersection is residually maximal and it is the unique irredundant decomposition of $A$ into irreducible ideals. Moreover, if $P_{i}$ is the adjoint prime of $A_{i}$, then $\left\{P_{i}\right\}_{i \in I} \subseteq$ $\mathcal{X}_{A}$.

Proof. Apply Theorem 5.1, Corollary 5.3 and Lemma 5.5.

Remark 5.7. Corollary [5.6 need not be true for rings that are not arithmetical. In particular, the uniqueness assertion of the corollary fails in the Noetherian case. For example, if $x$ is an indeterminate over a field $F$ and $(R, M)$ is the local domain $F\left[x^{2}, x^{3}\right]_{\left(x^{2}, x^{3}\right)}$, then $M^{2}=\left(x^{4}, x^{5}\right) R$ is $M$-primary and has an irredundant decomposition $M^{2}=x^{2} R \cap x^{3} R$, where $x^{2} R$ and $x^{3} R$ are irreducible. However, neither $x^{2} R$ nor $x^{3} R$ is an isolated component of $M^{2}$, since the only isolated component of $M^{2}$ is the ideal $M^{2}$ itself.

The uniqueness result of Theorem 5.1 suggests the question: What rings $R$ have the property that for every proper ideal $A$ of $R$ the canonical primal decomposition of $A$ given in (2) of Theorem 3.5 is an irredundant representation of $A$ as an intersection of irreducible isolated components? The rest of this section is devoted to a resolution of this question.

Theorem 5.8. The following statements are equivalent for a ring $R$.

(i) $R$ is an arithmetical ring.

(ii) For each proper ideal $A$ of $R, A=\bigcap_{P \in \mathcal{X}_{A}} A_{(P)}$ is a decomposition of $A$ into irreducible ideals $A_{(P)}$.

(iii) Each proper ideal $A$ of $R$ can be represented as an intersection of irreducible isolated components of $A$.

Proof. The implication (i) $\Rightarrow$ (ii) follows from Theorems 1.8 and 3.5; the implication (ii) $\Rightarrow$ (iii) is clear. To complete the proof it suffices to show that (iii) $\Rightarrow$ (i). To show $R$ is arithmetical, we establish that $R_{M}$ is a valuation ring for each maximal ideal $M$ of $R$. Let $M$ be a maximal ideal of $R$, and let $a, b \in M$. Define $I=(a, b) R$. Then by (iii), there is a collection $\mathcal{C}$ of prime ideals of $R$ such that $I M=\bigcap_{P \in \mathcal{C}}(I M)_{(P)}$, and each $(I M)_{(P)}$ is irreducible. If $M \notin \mathcal{C}$, then $(I M)_{(P)}=I_{(P)}$ for all $P \in \mathcal{C}$. But this implies

$$
I \subseteq \bigcap_{P \in \mathcal{C}} I_{(P)}=I M .
$$

Since $I$ is finitely generated, $I \nsubseteq I M$. It follows that $M \in \mathcal{C}$ and $(I M)_{(M)}$ is an irreducible ideal of $R$. As in Remark 1.6. $I M R_{M}$ is an irreducible ideal of $R_{M}$. Thus by the observation $(\dagger)$ made in the course of proving Theorem 1.8, either $R_{M} a \subseteq R_{M} b$ or $R_{M} b \subseteq R_{M} a$. This completes the proof.

Lemma 5.9. Let $R$ be a ring, and $A$ be an ideal of $R$ that has only finitely many minimal primes $P_{1}, P_{2}, \ldots, P_{n}$. If these minimal primes are comaximal, then each $P_{i}$ is a Zariski-Samuel associated prime of $A$. 
Proof. We may assume $n>1$. By assumption, there exist $a \in P_{1}$ and $b \in P_{2} \cdots P_{n}$ such that $a+b=1$. Moreover, $a b$ has some power $(a b)^{n}$ in $A$. Then $\left(a^{n}, b^{n}\right) R=R$ and $a^{n} \in A: b^{n}$. Hence $A: b^{n}$ has radical $P_{1}$, and $P_{1}$ is a Zariski-Samuel associated prime of $A$. Similarly, $P_{2}, P_{3}, \ldots, P_{n}$ are Zariski-Samuel associated primes of $A$.

Lemma 5.10. Let $R$ be a ring in which incomparable prime ideals are comaximal, for example an arithmetical ring. If $A$ is a radical ideal of $R$, then each $P \in \mathcal{X}_{A}$ is a minimal prime of $A$ and $A_{(P)}=P$. Thus the following are equivalent for $a$ radical ideal $A$ of $R$ :

(i) The representation of $A$ as the intersection of its minimal primes is irredundant.

(ii) The canonical primal decomposition of $A$ given in (2) of Theorem 3.5 is irredundant.

Moreover, these conditions hold for every radical ideal of $R$ if and only if every ideal of $R$ has only finitely many minimal primes.

Proof. Let $A$ be a radical ideal of $R$. Since incomparable prime ideals of $R$ are comaximal, each $P \in \mathcal{X}_{A}$ contains a unique minimal prime $Q$ of $A$. Since $A$ is a radical ideal, $A_{(P)} \subseteq A_{(Q)}=Q$ are radical ideals with $Q$ the unique minimal prime of $A_{(P)}$. Thus $A_{(P)}=Q$ and $Q=P$. Therefore conditions (i) and (ii) are equivalent.

It is clear that if every ideal of $R$ has only finitely many minimal primes, then conditions (i) and (ii) hold for each radical ideal of $R$. To prove the converse, let $A$ be a radical ideal of $R$ and let $\left\{P_{i}\right\}_{i \in I}$ be the set of minimal primes of $A$. By hypothesis, the representation $A=\bigcap_{i \in I} P_{i}$ is irredundant.

To prove Lemma 5.10 it suffices to prove the set $I$ is finite. We may assume that $I$ has cardinality at least two. For each $i \in I$, let $A_{i}=\bigcap P_{j}$, where $j \in I$ and $j \neq i$. The representation $A_{i}=\bigcap_{j \neq i} P_{j}$ is irredundant and each $P_{j}, j \neq i$, is a minimal prime of $A_{i}$. Since by hypothesis the representation of each radical ideal of $R$ as the intersection of its minimal primes is irredundant, it follows that $\left\{P_{j}: j \in I, j \neq i\right\}$ is the set of minimal primes of $A_{i}$.

We observe that $A_{i}$ and $P_{i}$ are comaximal. For if there exists a prime ideal $Q$ of $R$ with $A_{i}+P_{i} \subseteq Q$, then $Q$ must contain a minimal prime of $A_{i}$. But for $j \neq i$, $P_{j}$ and $P_{i}$ are distinct minimal primes of $A$ and thus are incomparable and hence by hypothesis comaximal. It follows that $\sum_{i \in I} A_{i}=R$. For if $\sum_{i \in I} A_{i} \subseteq Q$, where $Q$ is prime in $R$, then $A \subseteq Q$ implies $Q$ contains some minimal prime of $A$, so $Q$ contains $P_{i}$ for some $i \in I$. But $A_{i}$ and $P_{i}$ are comaximal, a contradiction. Thus $\sum_{i \in I} A_{i}=R$. Hence there exists a finite subset $J$ of $I$ such that $\sum_{i \in J} A_{i}=R$. We must have $J=I$, for by construction $\sum_{j \in J} A_{j} \subseteq P_{i}$ for $i \in I \backslash J$.

Remark 5.11. Without the hypothesis that incomparable prime ideals of $R$ are comaximal, it can happen that every radical ideal of $R$ is the irredundant intersection of its minimal primes and yet there exist radical ideals of $R$ having infinitely many minimal primes. This is illustrated by the $\operatorname{ring} R / A$ of Example 5.4. This ring is one-dimensional reduced with a unique maximal ideal and infinitely many minimal primes $P_{n} / A$. Every radical ideal of $R / A$ is the irredundant intersection of its minimal primes. Moreover, the zero ideal of $R / A$ is a primal radical ideal that has infinitely many minimal primes.

We remark that even in a 2-dimensional Prüfer domain it can happen that there exists a radical ideal $A$ with infinitely many minimal primes such that $A$ is the 
irredundant intersection of its minimal primes. Thus the hypothesis in Lemma 5.10 about every radical ideal is necessary. This is illustrated by the following example.

Example 5.12. Let $F$ be a field of characteristic different from 2 and let $x, y$ be indeterminates over $F$. Let $V_{11}$ be the discrete rank-one valuation domain obtained by localizing the polynomial ring $F[x, y]$ at the prime ideal generated by $y$. Then $V_{11}=F(x)+P_{11}$, where $P_{11}$ denotes the maximal ideal of $V_{11}$. Define $R_{1}=W_{11}$ to be the rank-2 valuation domain $F[x]_{x F[x]}+P_{11}$.

Let $K_{1}=F(x, y)$, and let $K_{2}$ be a finite algebraic extension field of $K_{1}$ in which the valuation domain $V_{11}$ has at least two extensions, $V_{21}$ and $V_{22}$. For example, one may take $K_{2}=K_{1}[z]$, where $z$ is a root of the polynomial $Z^{2}-y-1$. Then $V_{11}[z]$ is the intersection of two valuation domains of the field $K_{2}$ that extend $V_{11}$, which we may label as $V_{21}$ and $V_{22}$, where $z-1$ generates the maximal ideal of $V_{21}$ and $z+1$ generates the maximal ideal of $V_{22}$.

The valuation domains $V_{21}$ and $V_{22}$ are each localizations of the integral closure $\overline{W_{11}}$ of $W_{11}$ in the field $K_{2}$, say at prime ideals $Q_{21}$ and $Q_{22}$ of $\overline{W_{11}}$. By the GoingUp Theorem [25, (10.9)], there exist prime ideals $N_{21} \supset Q_{21}$ and $N_{22} \supset Q_{22}$ of $\overline{W_{11}}$ with $N_{21}$ and $N_{22}$ each lying over the maximal ideal of $W_{11}$. Hence by 32 , Theorem 13, page 31], there exist extensions $W_{21}$ and $W_{22}$ of the rank-2 valuation domain $W_{11}$ to the field $K_{2}$ such that $W_{2 i} \subset V_{2 i}, i=1,2$. Define $R_{2}=W_{21} \cap W_{22}$. By the Theorem of Independence of Valuations [25, (11.11)], we see that $R_{2}$ is a Prüfer domain with precisely 4 nonzero prime ideals, these prime ideals being $P_{21} \subset M_{21}$, contracted from $W_{21}$, and $P_{22} \subset M_{22}$, contracted from $W_{22}$.

Let $A_{1}$ be the ideal $P_{11}$ of $R_{1}$ and define the ideal $A_{2}$ of $R_{2}$ as $A_{2}=M_{21} \cap P_{22}$. Observe that $A_{2} \cap R_{1}=A_{1}$ and $\operatorname{Ass}\left(A_{2}\right)=\left\{M_{21}, P_{22}\right\}$, with $M_{21}$ and $P_{22}$ the minimal primes of $A_{2}$.

There exists a finite algebraic extension field $K_{3}$ of $K_{2}$ for which the valuation domain $V_{22}$ has at least two distinct extensions $V_{32}$ and $V_{33}$, see, for example [15. Lemma 1.3]. Let $V_{31}$ be an extension of $V_{21}$ to the field $K_{3}$. There exists an extension $W_{31}$ to $K_{3}$ of the valuation domain $W_{21}$ such that $W_{31} \subset V_{31}$. Also there exist extensions $W_{32}$ and $W_{33}$ of $W_{22}$ to $K_{3}$ such that $W_{3 i} \subset V_{3 i}, i=2,3$.

Define $R_{3}=W_{31} \cap W_{32} \cap W_{33}$. Again by [25, (11.11)], we see that $R_{3}$ is a Prüfer domain with precisely 6 nonzero prime ideals, these prime ideals being $P_{3 i} \subset M_{3 i}$ contracted from $W_{3 i}, i=1,2,3$. Define $A_{3}=M_{31} \cap M_{32} \cap P_{33}$. This representation of $A_{3}$ is irredundant, so $\operatorname{Ass}\left(A_{3}\right)=\left\{M_{31}, M_{32}, P_{33}\right\}$. Moreover, $A_{3} \cap R_{2}=A_{2}$.

Continuing in this way, we obtain by induction for each positive integer $n$ a finite algebraic extension field $K_{n}$ of $F(x, y)$ and a Prüfer domain $R_{n}=\bigcap_{i=1}^{n} W_{n i}$, where the $W_{n i}$ are rank-2 valuation domains on the field $K_{n}$ and where $R_{n}$ has precisely $2 n$ nonzero prime ideals, these prime ideals being $P_{n i} \subset M_{n i}$ contracted from $W_{n i}, i=$ $1, \ldots, n$. Define $A_{n}=\left(\bigcap_{i=1}^{n-1} M_{n i}\right) \cap P_{n n}$. This representation of $A_{n}$ is irredundant, so $\operatorname{Ass}\left(A_{n}\right)=\left\{M_{n 1}, M_{n 2}, \ldots, M_{n n-1}, P_{n n}\right\}$. Moreover, $A_{n} \cap R_{n-1}=A_{n-1}$.

Let $R=\bigcup_{n=1}^{\infty} R_{n}$ and $A=\bigcup_{n=1}^{\infty} A_{n}$. Also define $M=\bigcup_{n=1}^{\infty} M_{n n}$ and $P=$ $\bigcup_{n=1}^{\infty} P_{n n}$. Then $R$ is a 2-dimensional Prüfer domain. The maximal ideals of $R$ are $M$ and $M_{r}=\bigcup_{n=r}^{\infty} M_{n r}, r=1,2, \ldots$, while the nonzero nonmaximal prime ideals of $R$ are $P$ and $P_{r}=\bigcup_{n=r}^{\infty} P_{n r}, r=1,2, \ldots$ We have $A=\left(\bigcap_{r=1}^{\infty} M_{r}\right) \cap P$, with this intersection irredundant. Moreover, every element of $M$ is in some $M_{r}$. Therefore every element of $M$ is non-prime to $A$. Thus $M$ is a maximal prime divisor of $A$. However, the $M$-principal component $A_{(M)}$ of $A$ is $P$, so $M \notin \operatorname{Ass}(A)$. This completes the presentation of the example. 
In the proof of Theorem 5.14 it will be convenient to use the following terminology from [9] or [3, page 62]. An integral domain $R$ satisfies (\#) (introduced in $\S 4$ ) if the intersection $R=\bigcap_{M} R_{M}$, where $M$ ranges over the set of maximal ideals of $R$, is irredundant in the sense that if $M$ is any maximal ideal of $R$, then $R \neq \bigcap_{N \neq M} R_{N}$ when $N$ ranges over the set of all maximal ideals that are distinct from $M$. We say that $R$ satisfies (\#\#) if

(\#\#) $\quad$ every overring of $R$ satisfies (\#).

The relevance of condition (\#\#) in the present context is that a Prüfer domain satisfies (\#\#) if and only if for each prime ideal $P$ of $R$, there exists a finitely generated ideal $J$ of $R$ such that $J$ is contained in $P$ and the only maximal ideals of $R$ that contain $J$ are those that contain $P$ (Theorem 3 in [9]). See also [3] for a recent analysis of the conditions (\#) and (\#\#) in Prüfer domains.

Lemma 5.13. Let $R$ be an arithmetical ring such that for every prime ideal $P$ of $R$, there exists a finitely generated ideal $J$ contained in $P$ such that the only maximal ideals of $R$ containing $J$ are those that contain $P$. Then every ideal of $R$ has only finitely many minimal primes. In particular, if $R$ is a Prüfer domain satisfying (\#\#), then every ideal of $R$ has only finitely many minimal primes.

Proof. Let $A$ be a proper ideal of $R$. If $P$ is a minimal prime ideal of $A$, then by assumption there exists a finitely generated ideal $J$ of $R$ such that $J \subseteq P$ and the only maximal ideals of $R$ containing $A$ are those that contain $P$. Hence the ideal $P / A$ of the ring $R / A$ is the radical of a finitely generated ideal. By Theorem 1.6 in [10, it follows that $R / A$ has finitely many minimal prime ideals. Therefore $A$ has only finitely many minimal primes in $R$. The statement about Prüfer domains satisfying (\#\#) now follows from Theorem 3 of [9].

Theorem 5.14. The following statements are equivalent for a ring $R$.

(i) For each proper ideal $A$ of $R$, the decomposition $A=\bigcap_{P \in \mathcal{X}_{A}} A_{(P)}$ is irredundant and the components $A_{(P)}$ are irreducible ideals.

(ii) $R$ is an arithmetical ring such that for every prime ideal $P$ of $R$, there exists a finitely generated ideal $J$ contained in $P$ such that the only maximal ideals of $R$ containing $J$ are those that contain $P$.

(iii) $R$ is an arithmetical ring with Noetherian maximal spectrum.

In particular, the integral domains having these equivalent properties are precisely the Prüfer domains that satisfy (\#\#).

Proof. (i) $\Rightarrow$ (ii) First we observe that if $B$ is a proper ideal of $R$, then $R / B$ also satisfies (i). For if $A$ is a proper ideal of $R$ containing $B$, then by (i) the decomposition $A=\bigcap_{P \in \mathcal{X}_{A}} A_{(P)}$ is irredundant and each component $A_{(P)}$ is irreducible. It is straightforward to verify that for each $x \in R,(A: x) / B=A / B:_{R / B} x+B$, and from this it follows that $\operatorname{Ass}(A / B)=\{P / B: P \in \operatorname{Ass}(A)\}$; hence $\mathcal{X}_{A / B}=\{P / B$ : $\left.P \in \mathcal{X}_{A}\right\}$. Moreover, for each $P \in \mathcal{X}_{A}$ we have $(A / B)_{(P / B)}=A_{(P)} / B$, so that each $(A / B)_{(P / B)}$ is irreducible. Now by Theorem 3.5,

$$
A / B=\bigcap_{P \in \mathcal{X}_{A}}(A / B)_{(P / B)}=\bigcap_{P \in \mathcal{X}_{A}} A_{(P)} / B .
$$

Hence the irredundancy of the canonical primal decomposition of $A / B$ follows from the irredundancy of the canonical primal decomposition of $A$. Thus (i) holds for $R / B$. 
We now prove that (i) implies (ii). By Theorem 5.8, $R$ is an arithmetical ring and by Lemma 5.10 each ideal of $R$ has only finitely many minimal primes. Since a domain homomorphic image of an arithmetical ring is a Prüfer domain and since in an arithmetical ring with finitely many minimal primes each of the minimal primes is the radical of a finitely generated ideal, it suffices by our above observation that (i) passes to homomorphic images to prove that the condition in (ii) holds in the case where $R$ is a Prüfer domain. In this case, as noted above, the condition in (ii) is equivalent to showing $R$ satisfies (\#\#). Furthermore, to verify (\#\#), it is enough to prove that every fractional overring $S$ of $R$ (i.e. $S$ is an overring of $R$ such that $r S \subseteq R$ for some nonzero $r \in R$ ) satisfies (\#) (see, for example, Proposition 2.5 in [29]). Let $S$ be a fractional overring of $R$. Since $R$ is a Prüfer domain, each maximal ideal of $S$ is of the form $S P$ for some prime ideal $P$ of $R$. Let $\left\{S P_{i}\right\}$ denote the collection of maximal ideals of $S$, where each $P_{i}$ is a prime ideal of $R$. Using the fact that $R_{P_{i}}$ is a valuation domain, it is easy to see that $S_{S P_{i}}=R_{P_{i}}$, since $P_{i}$ survives in $S_{S P_{i}}$. We show that $S$ satisfies (\#) by verifying that for each $j$, the quasilocal ring $S_{S P_{j}}$ is relevant to the decomposition $S=\bigcap_{i} S_{S P_{i}}$.

Let $S P_{j}$ be a maximal ideal of $S$ and let $r$ be a nonzero element of $P_{j}$ such that $r S \subset P_{j}$. We use Proposition 2.8 to calculate $\mathcal{X}_{A}$, where $A=r S$. We show that $\mathcal{X}_{A}$ consists of the prime ideals in $\left\{P_{i}\right\}$ that contain $A$.

We first observe:

Claim 1. If $P \in\left\{P_{i}\right\}$ and $A \subseteq P$, then $P \in \operatorname{Ass}(A)$.

We have $\operatorname{End}\left(A_{P}\right)=\operatorname{End}\left(S_{P}\right)=R_{P}$. The first equality follows from the fact that $A$ is a principal ideal of $S$, and the second equality from the fact that $S \subseteq R_{P}$. By Proposition 2.8 $P \in \operatorname{Ass}(A)$. We next observe:

Claim 2. $\mathcal{X}_{A} \subseteq\left\{P_{i}\right\}$.

Let $Q \in \mathcal{X}_{A}$. By Proposition 2.8, $R_{Q}=\operatorname{End}\left(A_{M}\right)=S_{M}$ for some maximal ideal $M$ of $R$. Thus $Q S \neq S$, so $Q S \subseteq P S$ for some $P \in\left\{P_{i}\right\}$. Since $S$ is flat over $R$, we have $Q \subseteq P$. By Claim 1, $P \in \operatorname{Ass}(A)$. Since $Q \in \mathcal{X}_{A}$ is maximal in $\operatorname{Ass}(A)$, we have $Q=P$.

Since there are no inclusion relations among the prime ideals in $\left\{P_{i}\right\}$, it follows from Claims 1 and 2 that $\mathcal{X}_{A}=\left\{P \in\left\{P_{i}\right\}: A \subseteq P\right\}$.

By (i), the decomposition $A=\bigcap_{P \in \mathcal{X}_{A}} A_{(P)}$ is irredundant, and by an application of the pertinent definitions, this implies there exists $x \in R \backslash A$ such that $A: x=$ $A x^{-1} \cap R \subseteq P_{j}$ but $A x^{-1} \cap R \nsubseteq P_{i}$ for all $i \neq j$ such that $A \subseteq P_{i}$. Since $R$ is a Prüfer domain and $A=S r$, we have $A x^{-1} \cap S=S\left(A x^{-1} \cap R\right) \subseteq S P_{j}$, and if $A x^{-1} \cap S \subseteq S P_{i}$ for some $i \neq j$, then $A x^{-1} \cap R \subseteq S P_{i} \cap R=P_{i}$. If $A \subseteq P_{i}$, this is a contradiction to the choice of $x$. Also, since $A \subseteq A: x$, it cannot be the case that $A: x \subseteq P_{i}$ for any $i$ for which $A \nsubseteq P_{i}$. We conclude that the only maximal ideal of $S$ that contains $A:_{S} x=r S:_{S} x$ is $S P_{j}$. It follows that $r x^{-1} \in \bigcap_{i \neq j} S_{S P_{i}}$ but $r x^{-1} \notin S_{S P_{j}}$. Thus $S_{S P_{j}}$ is relevant to the decomposition $S=\bigcap_{i} S_{S P_{i}}$, and this proves that $S$ satisfies (\#). Consequently, $R$ satisfies (\#\#). This completes the proof that (i) implies (ii).

(ii) $\Longleftrightarrow$ (iii) The equivalence of (ii) and (iii) follows from [30, Corollaries 1.3 and 1.5].

(ii) $\Rightarrow$ (i) Let $A$ be a proper ideal of $R$. By Lemma 3.1, $A=\bigcap_{P \in \mathcal{X}_{A}} A_{(P)}$. We claim that this is an irredundant decomposition of $A$. Let $P \in \mathcal{X}_{A}$. By (ii), there exists a finitely generated ideal $J$ of $R$ that is contained in $P$ but in no maximal 
ideal of $R$ not containing $P$. Since $R$ is arithmetical, $P=\bigcup_{i} P_{i}$ for some chain of weak-Bourbaki associated primes of $A$. Since $J$ is finitely generated, there exists a weak-Bourbaki associated prime $P_{i}$ of $A$ such that $J \subseteq P_{i} \subseteq P$. It follows that $P_{i} \nsubseteq \subseteq$ for any $Q \in \mathcal{X}_{A}$ with $Q \neq P$. For $Q$ and $P$ distinct in $\mathcal{X}_{A}$ are comaximal and $J \subseteq P_{i}$ is contained in no maximal ideal of $R$ not containing $P$. By definition, $P_{i}$ is a minimal prime ideal of $A: x$ for some $x \in R \backslash A$. By Lemma 5.13, $A: x$ has only finitely many minimal primes, so Lemma 5.9 implies that there exists $y \in R \backslash A$ such that $P_{i}=\sqrt{(A: x): y}=\sqrt{A: x y}$. It follows that $A: x y \subseteq P$ but $A: x y \nsubseteq \subseteq Q$ for any $Q \in \mathcal{X}_{A}$ with $Q \neq P$. Hence $x y \in \bigcap_{Q \neq P} A_{(Q)}$. Since $A: x y \subseteq P$, it is case that $x y \notin A$, so that $x y \notin A_{(P)}$. Therefore $\bigcap_{Q \neq P} A_{(Q)} \not \subset A_{(P)}$, and we conclude that the decomposition $A=\bigcap_{P \in \mathcal{X}_{A}} A_{(P)}$ is irredundant.

Remark 5.15. A ring $R$ is reduced and satisfies (i) and (ii) of Theorem 5.8 if and only if $R$ is a finite direct product of Prüfer domains that satisfy (\#\#). If $R$ satisfies (i) and (ii) of Theorem 5.14 then by Lemma 5.13, $R$ has finitely many minimal prime ideals. Since $R$ is reduced, (0) is the intersection of finitely many prime ideals, and since $R$ is arithmetical, these prime ideals must be comaximal. Consequently, $R$ is a finite direct product of Prüfer domains, each of which satisfies (\#\#). Conversely, it is not hard to verify that if $R$ is a finite direct product of Prüfer domains satisfying (\#\#), then $R$ is a reduced ring that satisfies (ii) of Theorem 5.14.

Remark 5.16. An arithmetical ring $R$ has Noetherian prime spectrum if and only if $R$ satisfies condition (ii) (or (i)) of Theorem 5.14 and $R$ has the ascending chain condition on prime ideals. This follows, for example, from Theorem 1.6 in [10] and the equivalence of (i) and (iii) of Lemma 1.5. In particular, an arithmetical ring $R$ of finite Krull dimension satisfies (ii) of Theorem[5.14 if and only if $R$ has Noetherian prime spectrum. On the other hand, it is easy to exhibit infinite-dimensional Prüfer domains satisfying (ii) of Theorem 5.14 that do not have Noetherian prime spectrum. For example, if $V$ is a valuation domain with value group $\bigoplus_{i=1}^{\infty} \mathbb{Z}$ given the reverse lexicographic order, then the maximal ideal of $V$ is the union of all the prime ideals properly contained in it.

Questions 5.17. We close with the following questions.

(1) In Theorem 5.14, we have characterized the rings $R$ such that for each proper ideal $A$ of $R$ the canonical primal decomposition of $A$ is irredundant with irreducible components. It would be interesting to classify the rings $R$ having the property that for every proper ideal $A$ of $R$ there exists a subset $C$ of $\operatorname{Ass}(A)$ such that $A=\bigcap_{P \in C} A_{(P)}$ is irredundant and each $A_{(P)}$ is irreducible. In view of Theorem [5.8, a ring with this property is an arithmetical ring, so the question is asking to classify the arithmetical rings $R$ for which each proper ideal $A$ can be written as an irredundant intersection $A=\bigcap_{P \in C} A_{(P)}$ for some subset $C$ of $\operatorname{Ass}(A)$. There exist Prüfer domains with this property that do not satisfy the equivalent conditions of Theorem [5.14] For example, if $R$ is the almost Dedekind domain that is not Dedekind of Example 2.2 of [14, pages 277-278], then $R$ does not satisfy the equivalent conditions of Theorem [5.14, but every proper ideal $A$ of $R$ is an irredundant intersection $A=\bigcap_{P \in C} A_{(P)}$ for some subset $C$ of $\operatorname{Ass}(A)$.

(2) What Prüfer domains $R$ have the property that for each proper Zariski closed subset $V$ of Spec $R$ there exists an ideal $A$ of $R \operatorname{such}$ that $\operatorname{Ass}(A)=$ 
$V$ ? If there exists a finitely generated ideal $A$ such that $V=\{P \in \operatorname{Spec} R$ : $A \subseteq P\}$, then Proposition 2.8 implies that $\operatorname{Ass}(A)=V$.

(3) Let $R$ be an arithmetical ring. Is it possible to characterize the posets $\operatorname{Ass}(A)$ of $\operatorname{Spec} R$ as $A$ varies over the ideals of $R$ ? By Proposition 2.7 if $P \in \operatorname{Ass}(A)$ and $Q \in \operatorname{Spec} R$ with $A \subseteq Q \subseteq P$, then $Q \in \operatorname{Ass}(A)$.

(4) In [6] we investigate for an ideal $A$ of an arithmetical ring the relationship between the set $\operatorname{Max}(A)$ of maximal prime divisors of $A$ and the set $\mathcal{X}_{A}$ of maximal members of the set of Krull associated primes of $A$. In this connection, it would be interesting to characterize the subsets $C$ of the set of maximal ideals of an arithmetical ring (or Prüfer domain) $R$ such that $C$ is the set of maximal prime divisors of an ideal $A$ of $R$.

\section{ACKNOWLEDGEMENT}

We thank Gabriel Picavet and David Rush for helpful conversations on the topics of this paper, and for showing us the connections of our work to the literature on Krull associated primes.

\section{REFERENCES}

1. R. Berger, Various notions of associated prime ideals, Annal. Univer. Saraviensis 5 (1994), 245-271. MR.95g:13001

2. N. Bourbaki, Commutative Algebra, Chapters 1 - 7, Springer-Verlag, 1989. MR90a:13001

3. M. Fontana, J. Huckaba and I. Papick, Prüfer domains, Marcel Dekker, 1998. MF.98d:13021

4. L. Fuchs, A condition under which an irreducible ideal is primary, Quart. J. Math. Oxford 19 (1948), 235-237. MR 10:280c

5. L. Fuchs, On primal ideals, Proc. Amer. Math. Soc. 1 (1950), 1-6. MR11:310d

6. L. Fuchs, W. Heinzer and B. Olberding, Maximal prime divisors in arithmetical rings, to appear in Venezia 2002 Proceedings, Lecture Notes in Pure and Applied Math., Marcel Dekker.

7. L. Fuchs and E. Mosteig, Ideal theory in Prüfer domains: an unconventional approach, J. Algebra 252 (2002), 411-430. MR2003e:13028

8. R. Gilmer, A counterexample to two conjectures in ideal theory, Amer. Math. Monthly, 74 (1967), 195-197.

9. R. Gilmer and W. Heinzer, Overrings of Prüfer domains II, J. Algebra 7 (1967), 281-302. MR 36:150

10. R. Gilmer and W. Heinzer, Primary ideals with finitely generated radical in a commutative ring, Manuscripta Math. 78 (1993), 201-221. MR.93k:13003

11. R. Gilmer and W. Heinzer, Imbeddability of a commutative ring in a finite-dimensional ring, Manuscripta Math. 84 (1994), 401-414. MR95i:13004

12. W. Heinzer, Quotient overrings of integral domains, Mathematika 17 (1970), 139-148. MR 42:244

13. W. Heinzer and D. Lantz, The Laskerian property in commutative rings, J. Algebra 72 (1981), 101-114. MR83h:13025

14. W. Heinzer and J. Ohm, Locally Noetherian commutative rings, Trans. Amer. Math. Soc., 158 (1971), 273-284. MR 43:6192

15. W. Heinzer and S. Wiegand, Prime ideals in two-dimensional polynomial rings, Proc. Amer. Math. Soc., 107 (1989), 577-586. MR90b:13010

16. J. Iroz and D. Rush, Associated prime ideals in non-Noetherian rings, Can. J. Math. 36 (1984), 344-360. MR85j:13002

17. C. Jensen, Arithmetical rings, Acta Math. Acad. Sci. Hung. 17 (1966), 115-123. MR 32:7577

18. I. Kaplansky, Commutative Rings (University of Chicago Press, 1974). MR49:10674

19. W. Krull, Idealtheorie in Ringen ohne Endlichkeitsbedingung, Math. Ann. 101 (1929), 729744.

20. W. Krull, Idealtheorie, Ergebnisse d. Math. (Springer-Verlag, Berlin, 1935). MR 37:5197 (2nd ed.) 
21. R. Kuntz, Associated prime divisors in the sense of Krull, Can. J. Math. 24 (1972), 808-818. MR 47:1788

22. D. McQuillan, Rings of integer-valued polynomials determined by finite sets, Proc. Royal Irish Acad. 85 (1985), 177-184. MR87g:13016

23. S. Mori, Über eindeutige Reduktion in Ringen ohne Teilerkettensatz, J. Sci. Hiroshima Univ. A 3 (1933), 275-318.

24. M. Nagata, Some remarks on prime divisors, Memoirs College of Science, Kyoto University, Series A, 33 (1960) 297-299. MR22:9518

25. M. Nagata, Local Rings (Interscience, 1962). MR27:5790

26. N. Nakano, Idealtheorie in einem speziellen unendlichen algebraischen Zahlkörper, J. Sci. Hiroshima Univ. Ser. A 16 (1953), 425-439. MR 15:510b

27. E. Noether, Idealtheorie in Ringbereichen, Math. Ann. 83 (1921), 24-66.

28. J. Ohm and P. Pendleton, Rings with Noetherian spectrum, Duke Math. J. 35 (1968), 631640. MR 37:5201

29. B. Olberding, Globalizing local properties of Prüfer domains, J. Algebra 205 (1998), 480-504. MR2000f: 13040

30. D. Rush and L. Wallace, Noetherian maximal spectrum and coprimely packed localizations of polynomial rings, Houston J. Math. 28 (2002) 437-448.

31. D. Underwood, On some uniqueness questions in primary representations of ideals. J. Math. Kyoto Univ. 9 (1969), 69-94. MR 40:134

32. O. Zariski and P. Samuel, Commutative Algebra, Vol. 2, Van Nostrand, Princeton 1960. $\operatorname{MR} 22: 11006$

Department of Mathematics, Tulane University, New Orleans, Louisiana 70118

E-mail address: fuchs@tulane.edu

Department of Mathematics, Purdue University, West Lafayette, Indiana 47907

E-mail address: heinzer@math.purdue.edu

Department of Mathematical Sciences, New Mexico State University, Las Cruces, NeW MeXico 88003-8001

E-mail address: olberdin@emmy.nmsu.edu 